\title{
Rate Constants for the $\beta$-Elimination of Tosyl Radical from a Variety of Substituted Carbon-Centered Radicals
}

\author{
Vitaliy I. Timokhin, ${ }^{\dagger \dagger}$ Stéphane Gastaldi, ${ }^{\dagger}$ Michèle P. Bertrand, ${ }^{*}{ }^{\dagger}$ \\ and Chryssostomos Chatgilialoglu
}

Laboratoire de Chimie Moléculaire Organique - UMR 6517, Boite 562 - Faculté des

Sciences St Jérôme, Université d'Aix-Marseille III, Av. Escadrille Normandie-Niemen, 13397

Marseille Cedex 20, France, and ISOF, Consiglio Nazionale delle Ricerche, Via P. Gobetti

$$
\text { 101, } 40129 \text { Bologna, Italy }
$$

\section{Supporting Information}

Table of Contents :

${ }^{1} \mathrm{H},{ }^{13} \mathrm{C}$ and DEPT NMR spectra data of coumpounds $\mathbf{2 a}, \mathbf{2 a}, \mathbf{2 b - 2 e , ~ 5 a - 5 e . . . . . . . . . . . . S 2 - S 3 4 ~}$

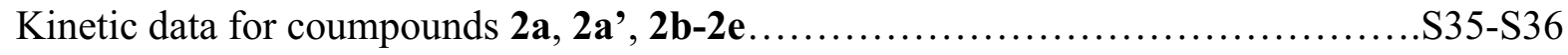




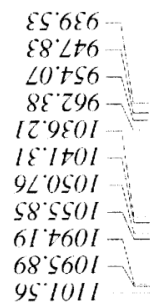

$6 Z 9 \mathcal{E} \mathrm{E} I-$

$\varepsilon \varepsilon z+\varepsilon l$

$60^{\circ} \mathrm{ctcl}$

lt thel -

$\varepsilon+\angle t \varepsilon l-$

IS $60 \mathrm{EI}$

otiosel

zt t58l-1

$t \angle S S E I$

OS 9581

$t+2581$

$\varepsilon \angle 2981$ -

E209I2-

SSIOIZ

$0+\angle 9 I 2$

62.6912

$5 S 8 \angle 12-$

68.2812

(582812

62.9812

$\angle L 1612-$

$\angle t^{\circ} \varepsilon 6 / 2$

$21.20 z \bar{c}-1$

$+8012 z$

ot $\varepsilon \varepsilon \varepsilon z$

$85686-$

$88 \angle t 6-$

$20+56-$

82296

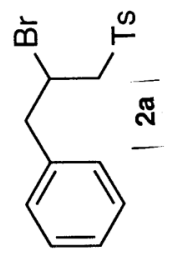

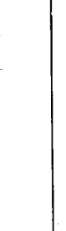
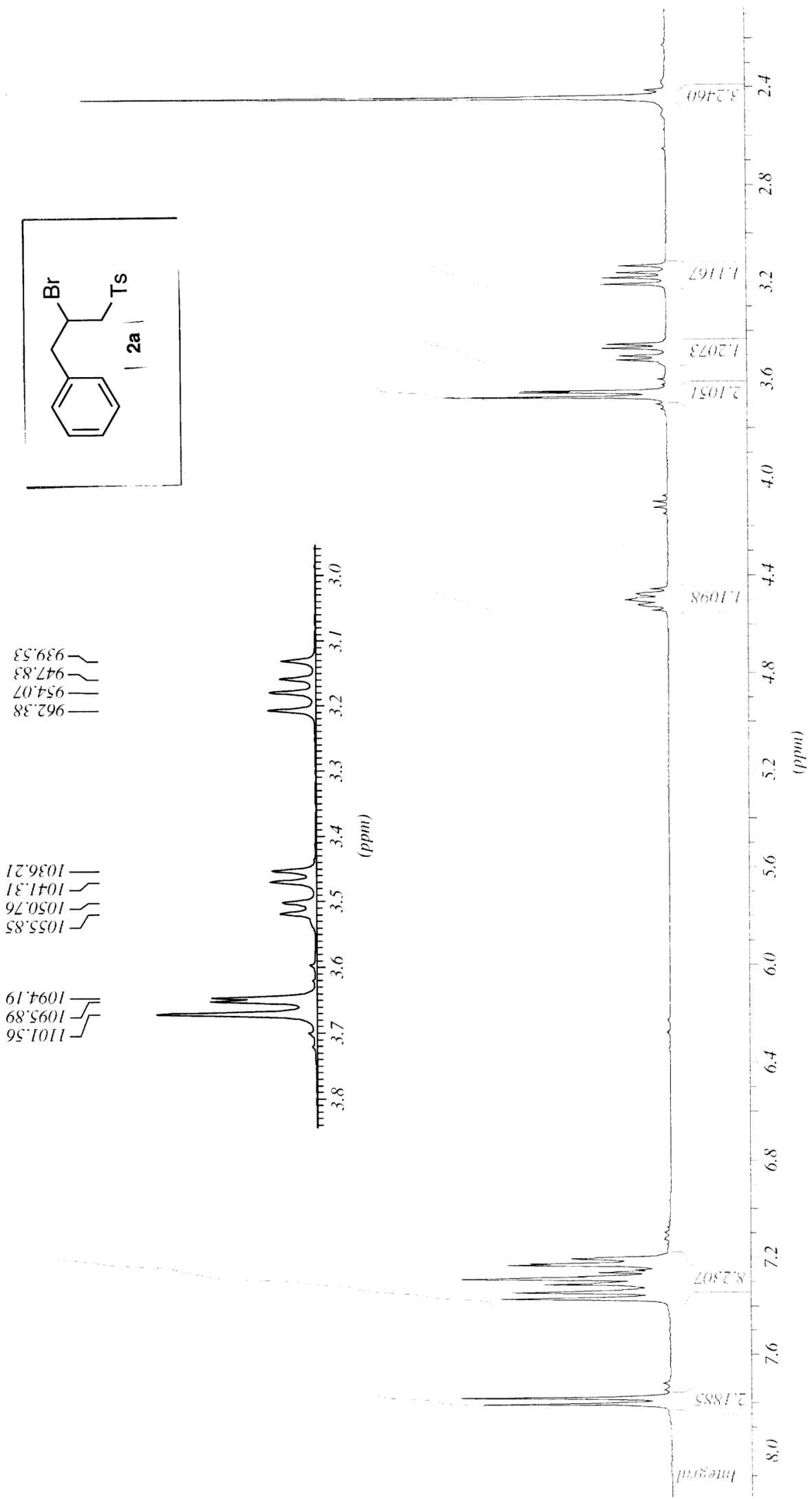

ミ

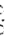


S3

$01112 z-$
$619 s \cdot 52-$

$18 \varepsilon 9^{\circ} t t$
$\operatorname{sol} 2 \mathrm{C} t$

$98 \angle Z \mathcal{E}^{\circ}-$
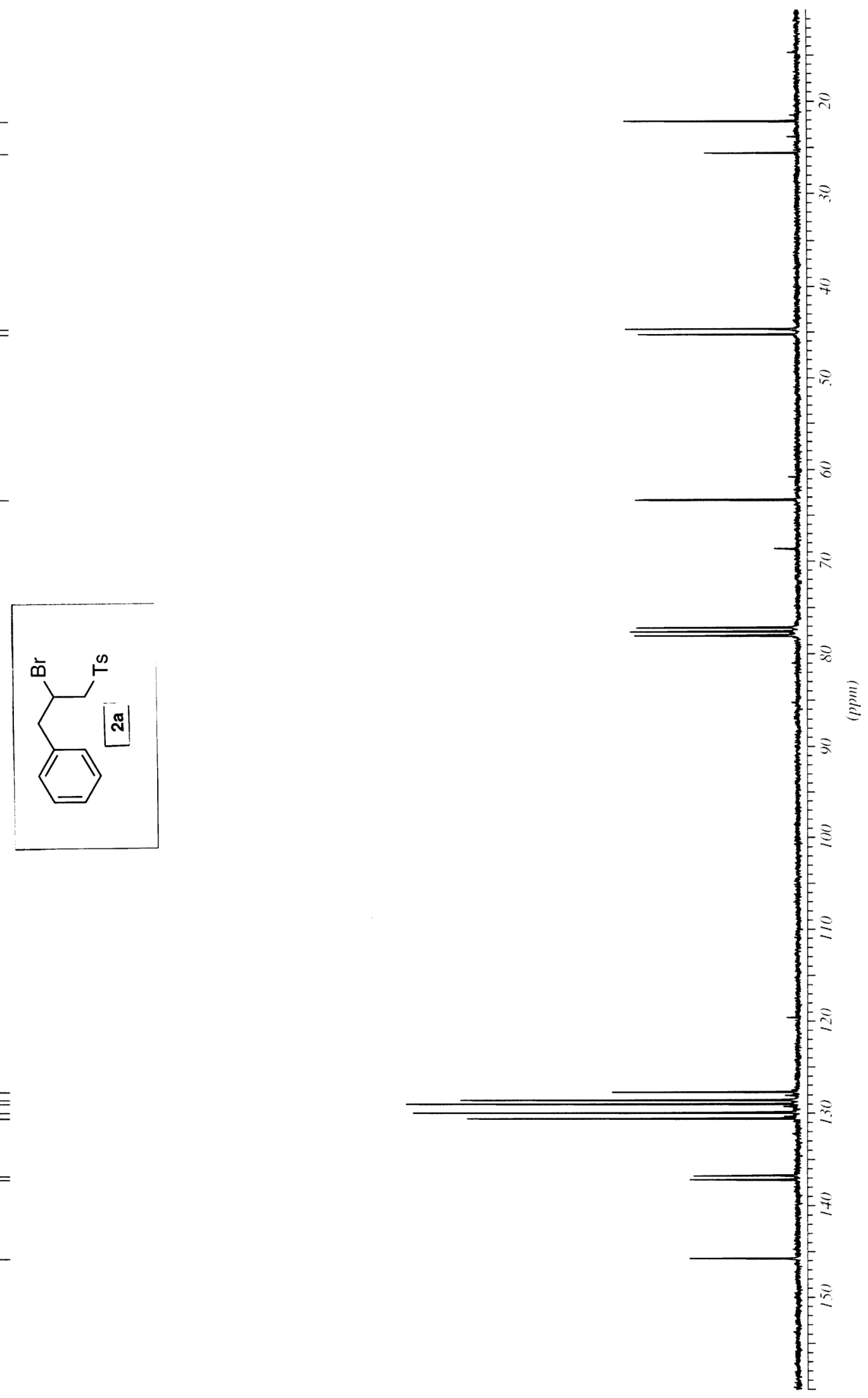

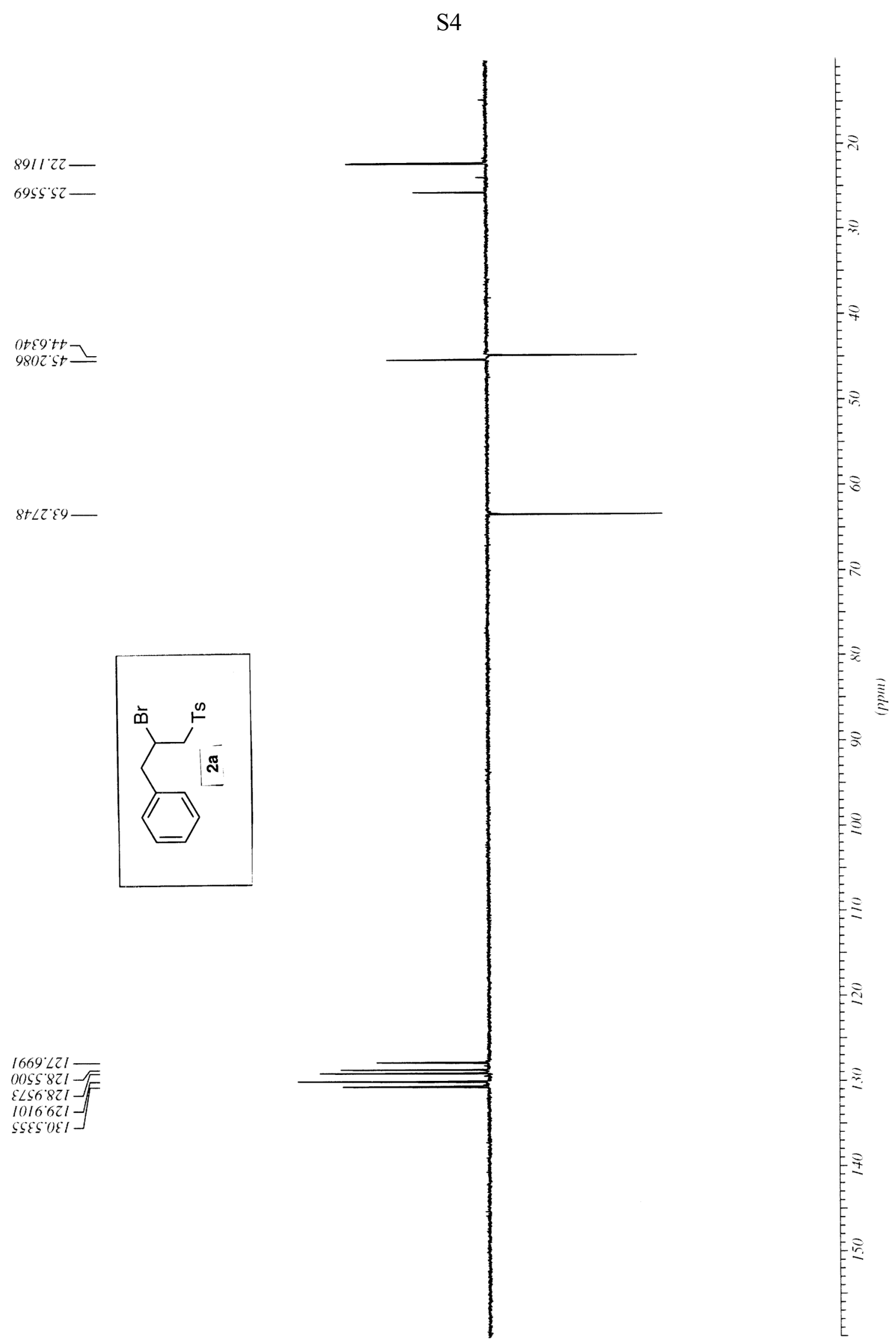

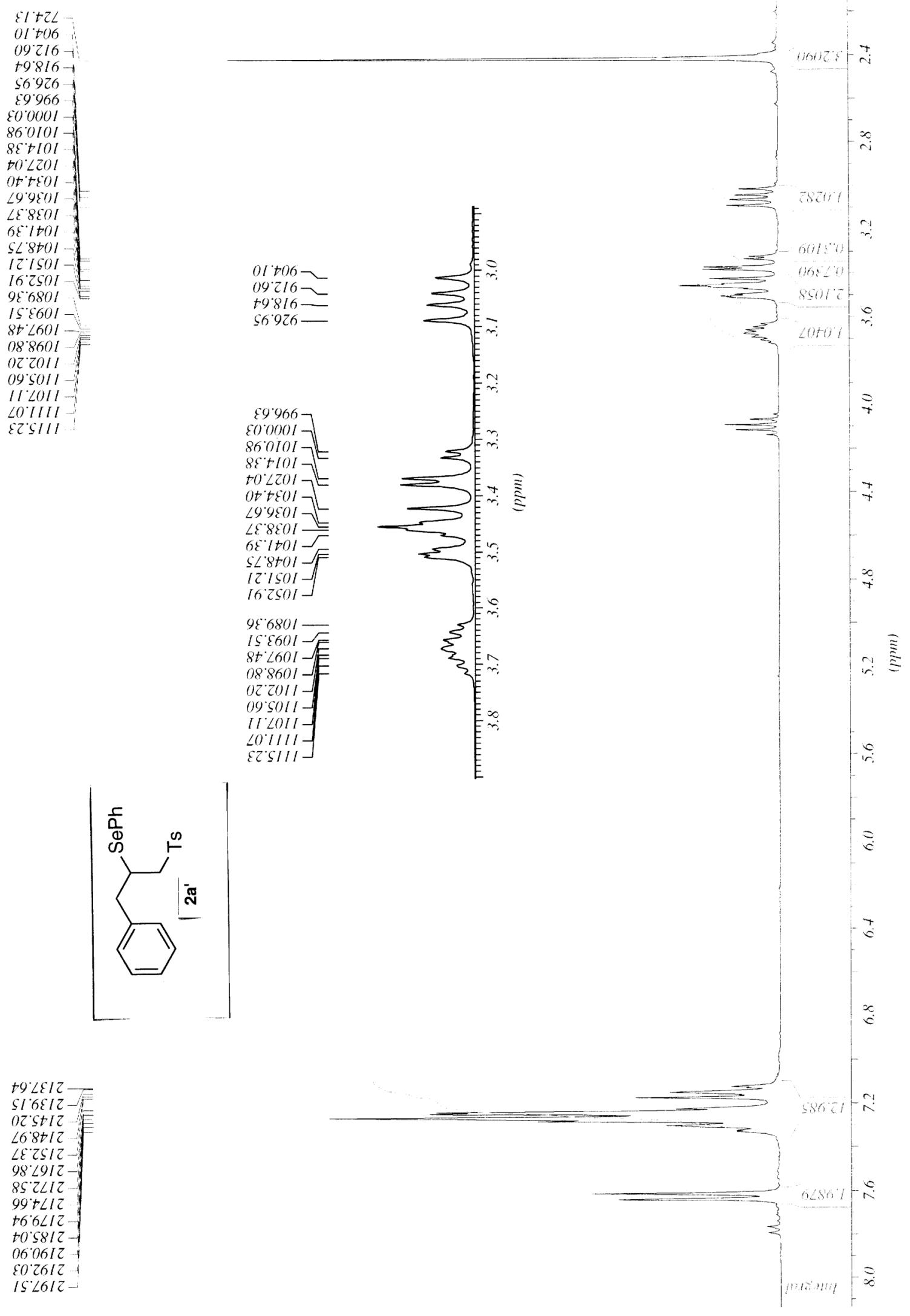

$+9 . \angle E I Z=$

$S I .6 \varepsilon I Z-$
$0 Z S+I z$

268 t 12

$\angle \varepsilon z S 12-$

$98^{\circ} \angle 912$

$8 s^{\circ} z \angle I Z$

$99^{\circ}+12$

$+6.6<12-$

to.58/2-

$0606 \mathrm{IZ}$

g0.26I2

$15 \angle 612$ -

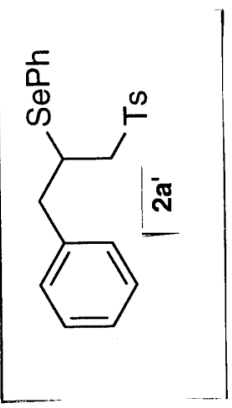

$19816=$ 


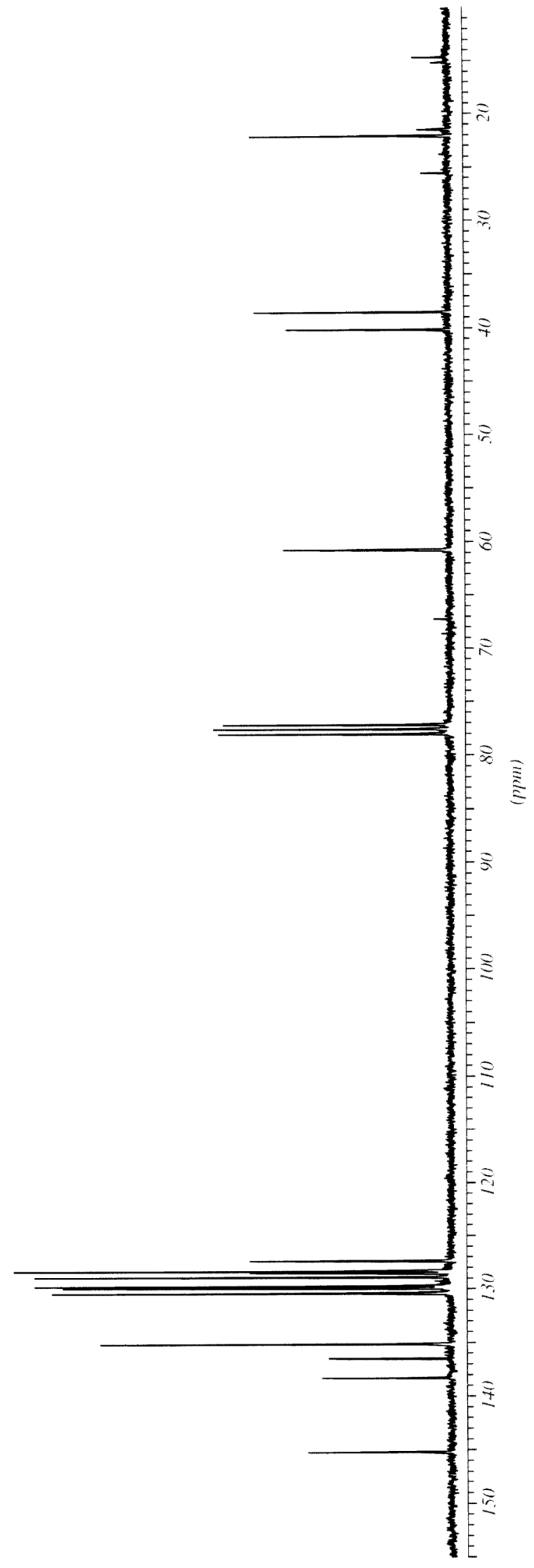

$Z \mid \angle E \angle Z I$

6828.821

$880582 I$

$0668.821-$ F

SLZL'62I -

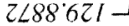

$\left.9110^{\circ} 0 \mathrm{El}\right]$

$\angle t S I S E L-T$

$8918891-$

$t \varepsilon O Z \mathcal{S} t-$

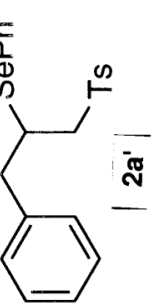


$\angle t 59^{\circ}+1$
$\varepsilon 6+1.51$

EI6t 12

$\angle \angle 80^{\circ} \mathrm{ZZ}-$

$2+95^{\circ} s 2$

(1)28588-
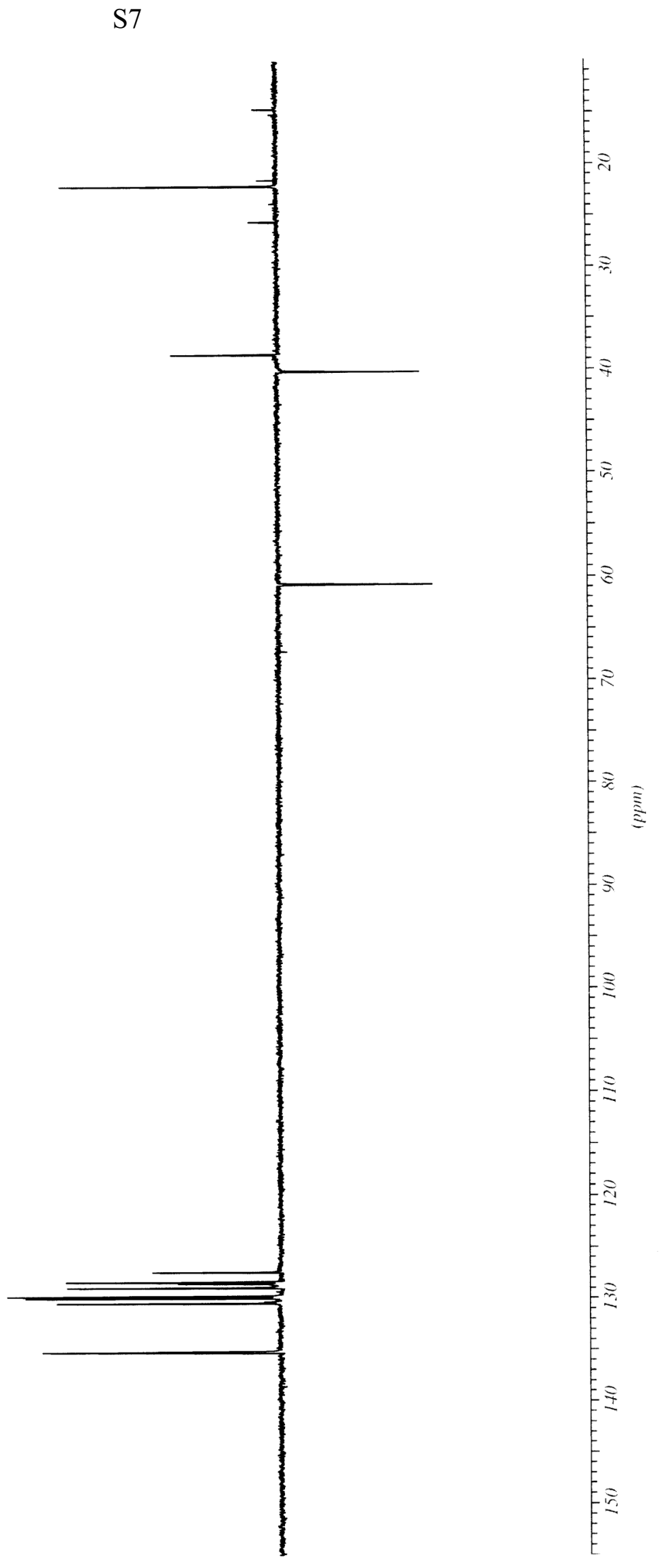

$8 I \angle E \angle Z I$

8189.821 - F

t905 821

क्ष

$282 \angle 621-$

$\$ \$ 68^{\circ} 621$

t9920 02

$61 \mathrm{I}^{\circ} \mathrm{O} \mathcal{E} \mathrm{I}-$

$628 I^{\circ} \mathcal{E E I}$

$6 \mathcal{S} I S E I$ 
$21+2 z-$

chore

$\angle I I S Z$
$26 \angle S Z$
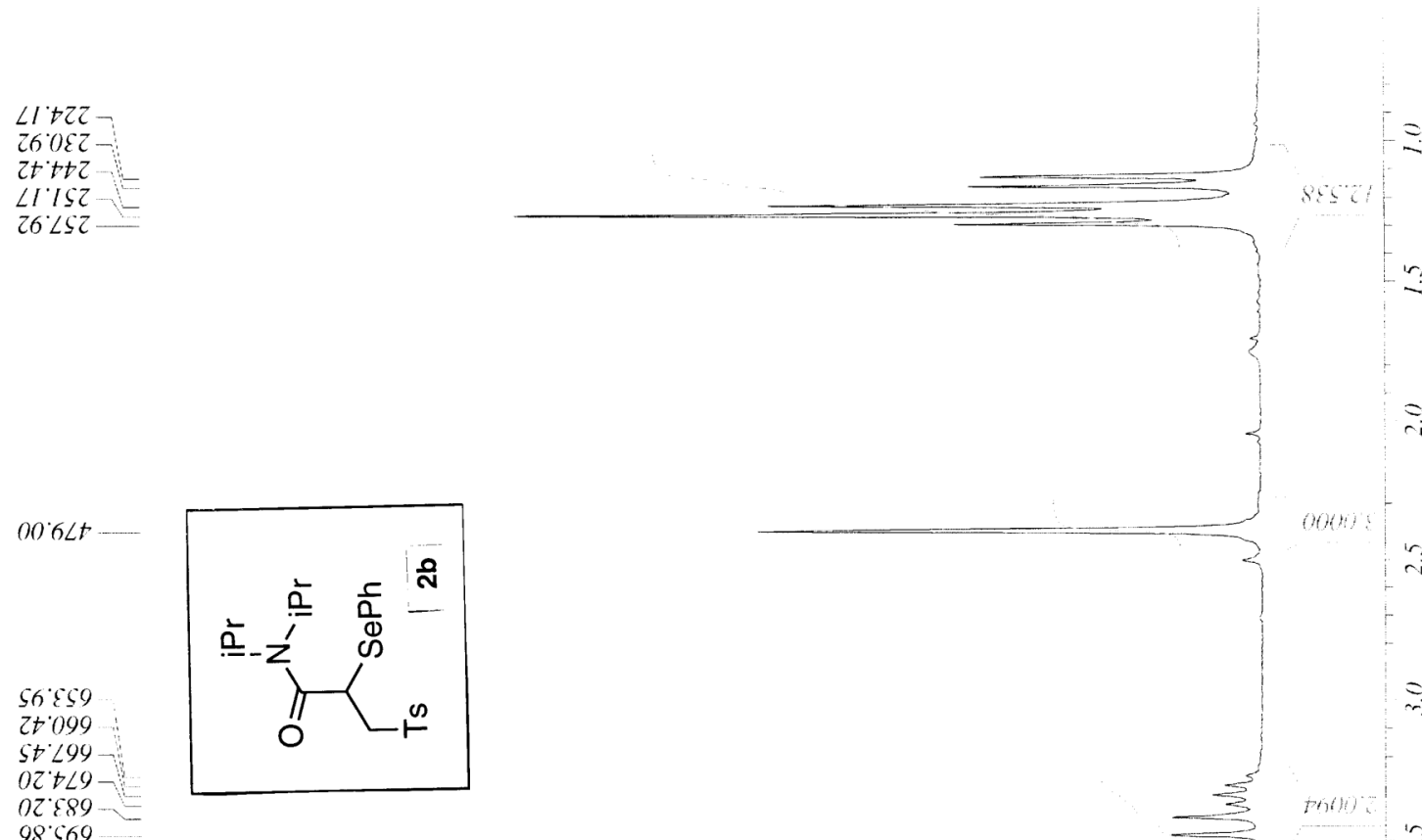

$80 \angle 18$

E8. $278-$

oध 088

50.288

$55658-$

$250 \angle 8-$

$+\angle C+\angle$

89.288

46.59

ct 099

st. $\angle 997 L$

$02+\angle 9$

$02 \cdot 889-$

98.569
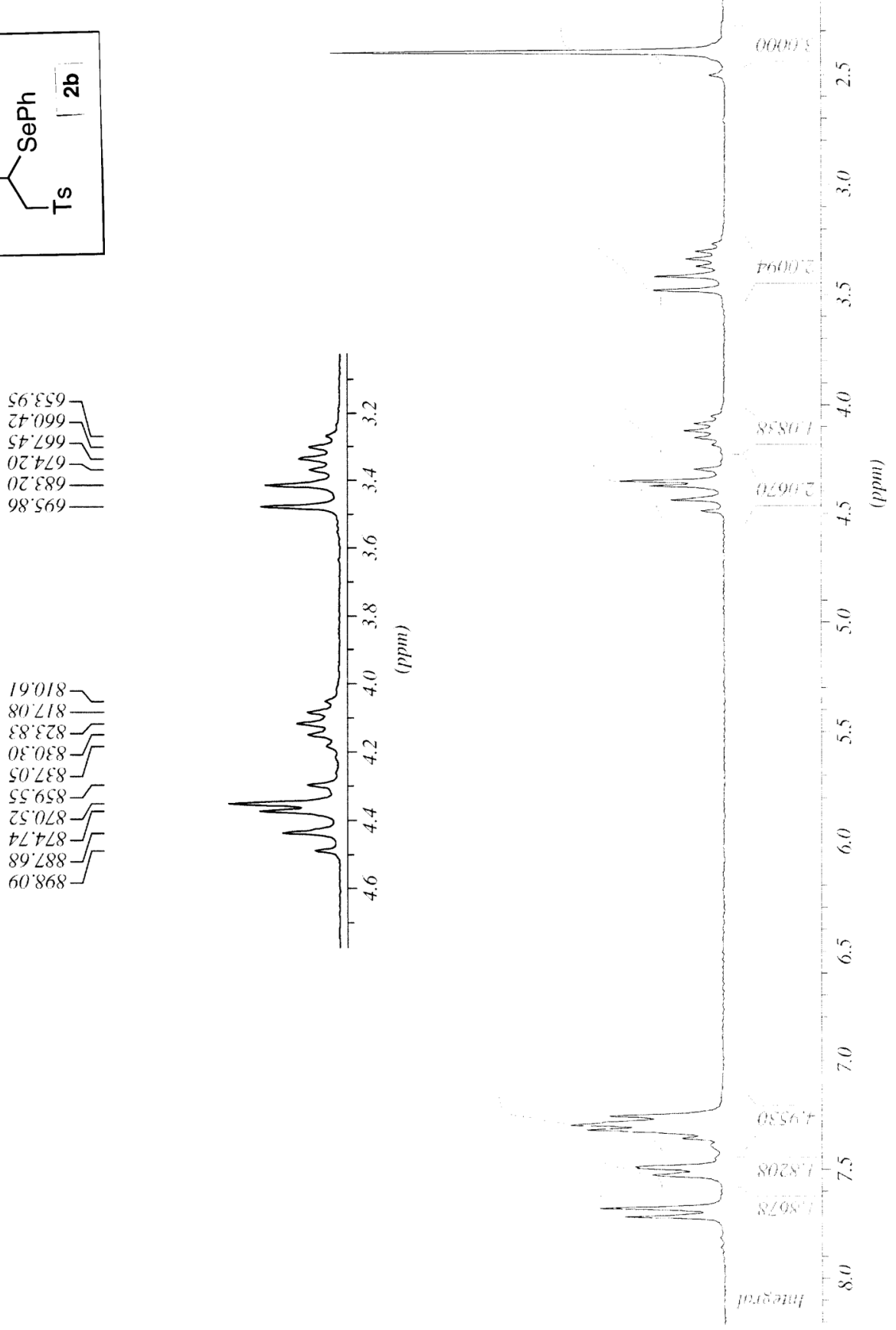

$90 \% 15+1$

$2 z \circ 65+1$

to $\varepsilon 9+l$

$\angle t: \angle 6 t 1$

$88.86+1$

$\angle O O^{\circ} O S I-$

$2 \angle S \& S I$

$88^{\circ} \mathrm{E}+\mathrm{S} /$ 
$2+5002$

258202

$829802-L$

$+18+9 \varepsilon-$

$9512 \cdot 9 t$

80zt'6t-

$1+5565$

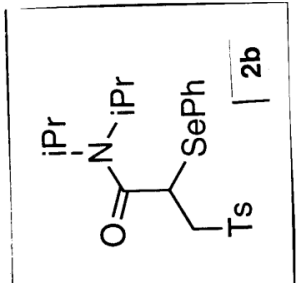

$+600821$

$0680621-$

$\angle \angle L E G Z I-$

$0985581=$
$5+68.981-$

$1+29+11$

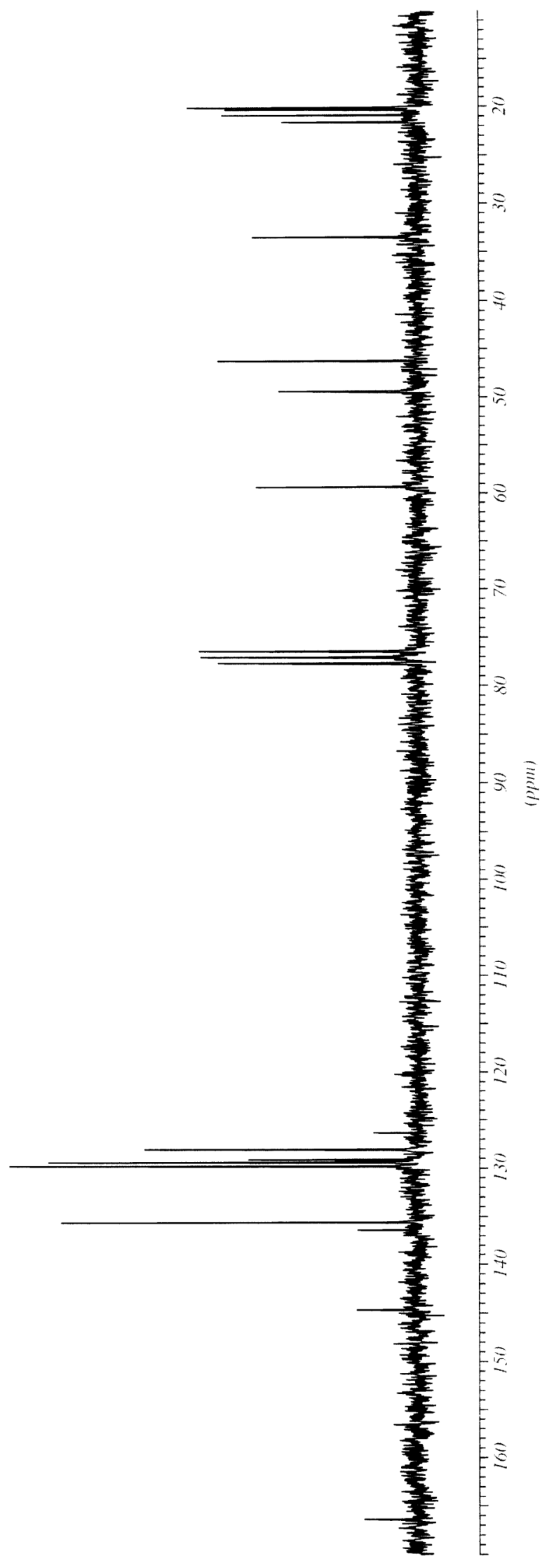

$\$ \angle 99^{\circ} 991$ 
$\varepsilon 9 \varepsilon 9^{\circ} 0 z$

EL98.0Z

$\angle 99122$ 느

$5890+5-$
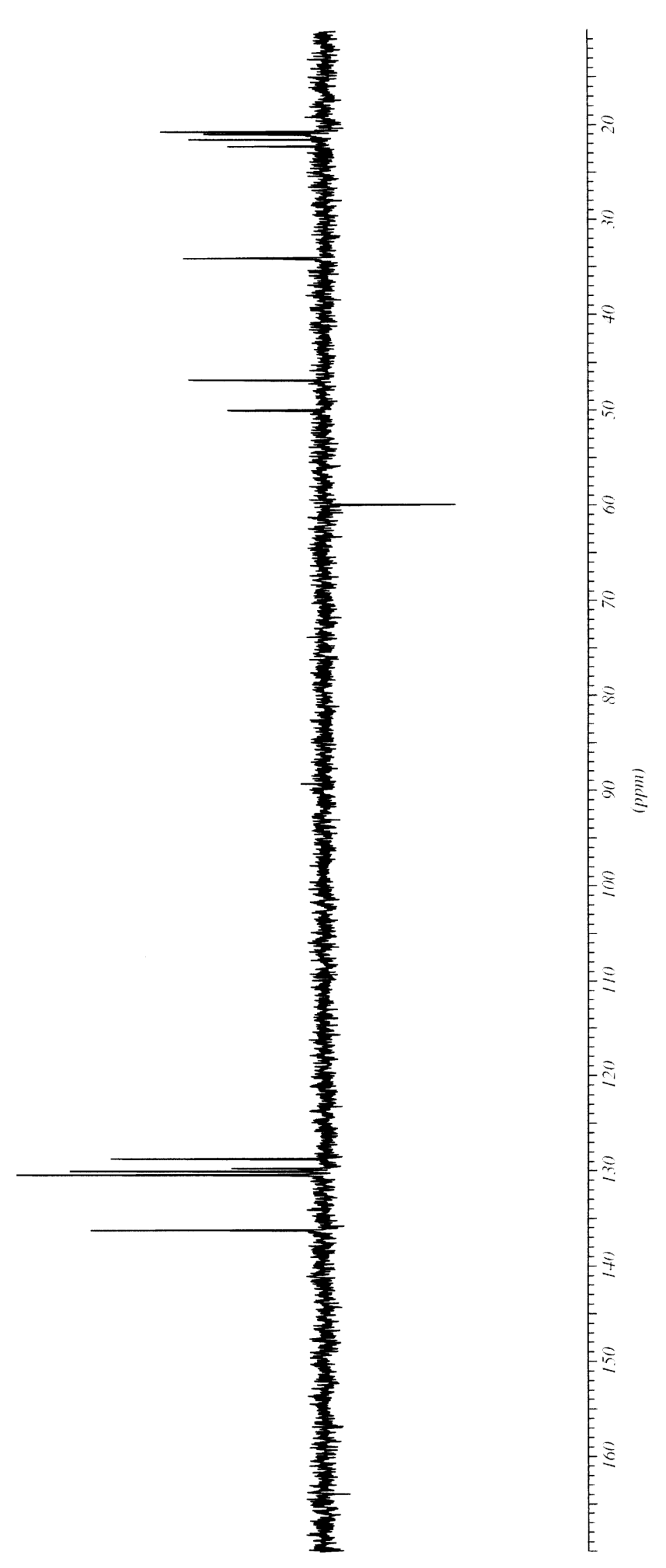

9IE9:82I

IIL962I-

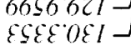

$\prod_{0}^{i} \sum_{\infty}^{\infty}$

2891981 - 
$+9+221$

$\varepsilon \angle 6 z 21$

$\angle \varepsilon 6 \varepsilon 21$

$8 z+t z l$

880921

$510<21]$

$\angle 8+\angle 21$

$88.68 \pi 1$ 는

95.2591

99.2591

$\angle S 2991$

$80^{\circ} \angle 991$ 느

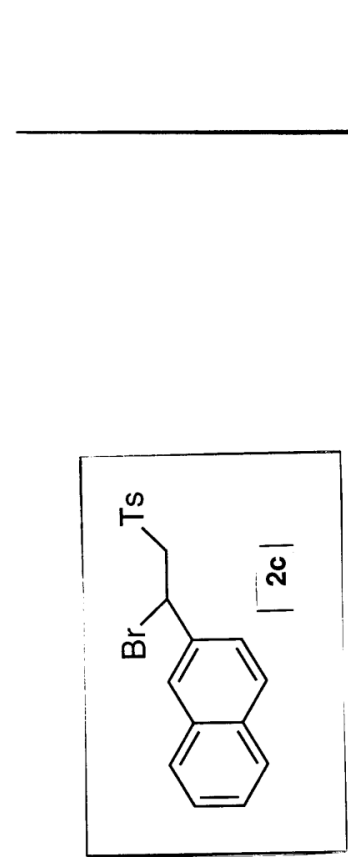

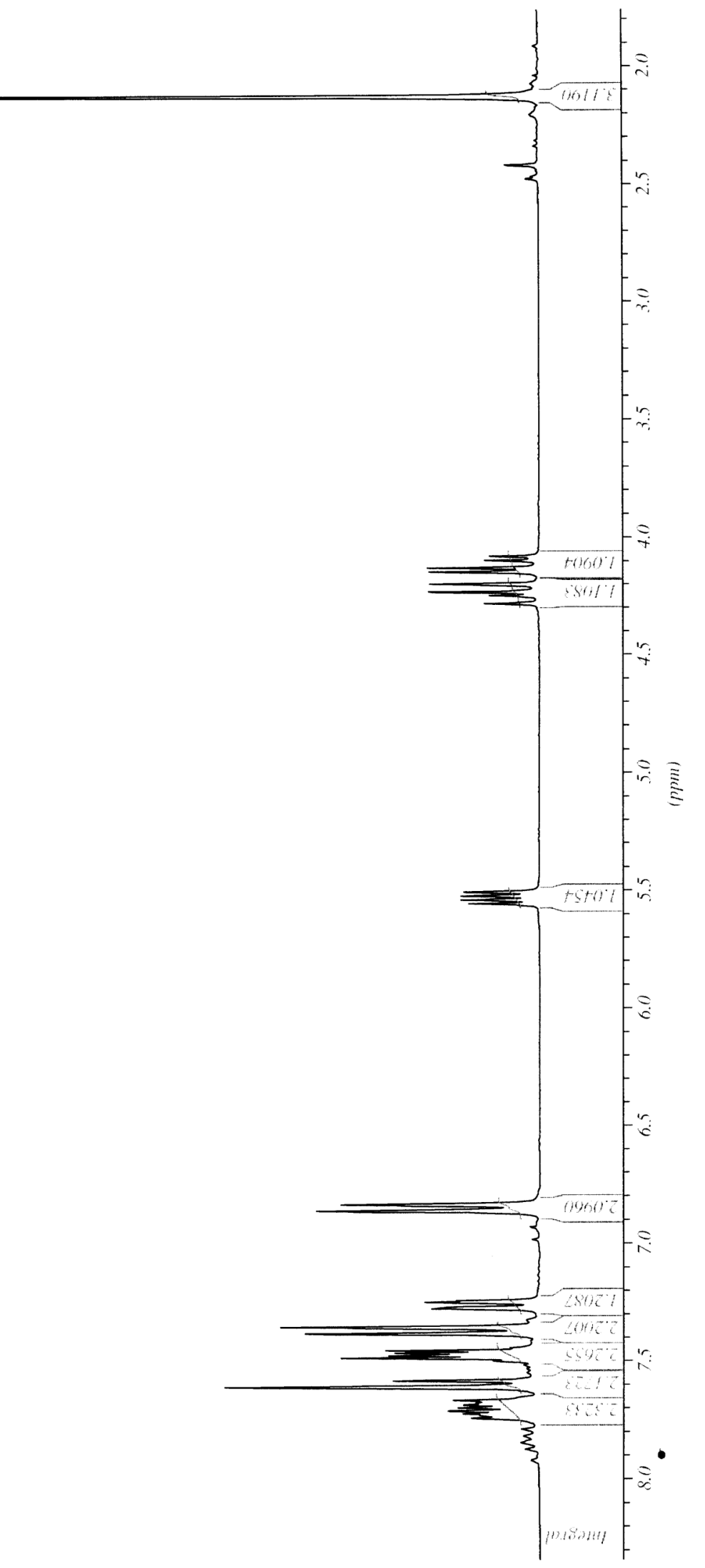

$8 \angle ' I S O Z$

$06.6502 \longrightarrow$

$6 S^{\circ} \varepsilon \angle 1 Z 7$

$98^{\circ} S \angle I Z-7$

60.2812

64.8812

$85 \angle 0 Z 2$

$68^{\circ} \mathrm{SIzZ}-\sqrt{\mathrm{e}}$

$20+5 z z$

zt $\angle \varepsilon z z-$

$28^{\circ} 0+22-=$

$78^{\circ} \mathrm{etz}-$

$98^{\circ} 9+22-$

$+90522-$

$+6{ }^{\circ} S \angle 2 Z-$

tot 822

$\angle 800 E 2-$

$\varsigma 9^{\circ}+0 \varepsilon z-$ 


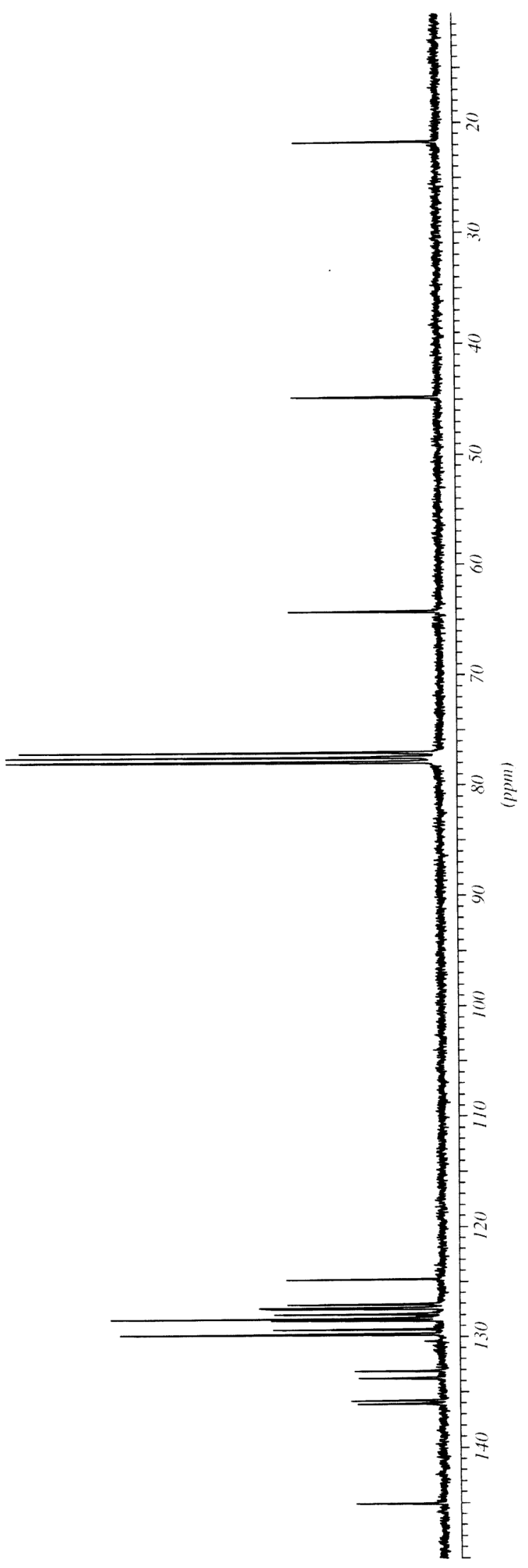

$+56<+21$

$0+00 \angle 2 I$
$0890^{\circ}<2 I$

9898221

$5816^{\circ} \mathrm{L2I}$

6825821

$296+821-F$

$\angle \mathrm{DCE} 62 \mathrm{Cl}$

$959 \angle 621$

$96 \angle 0 \mathcal{E \varepsilon l}-$

I8IL' $\mathrm{ESI}$

OSLL $S E 1-$

$06505+1$

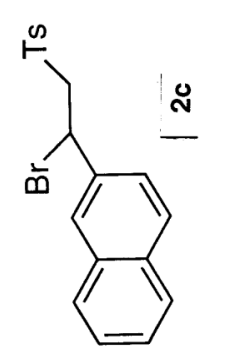




$$
1
$$


S14

1298012

$60^{\circ} 0501=$

898511 ح

ஹू

$6068 z 2$

$1+0 t z 2-$
$0 t \cdot t 2 z-1$

t 5 s $8+22-$

$62.622-$

O9.10\&2

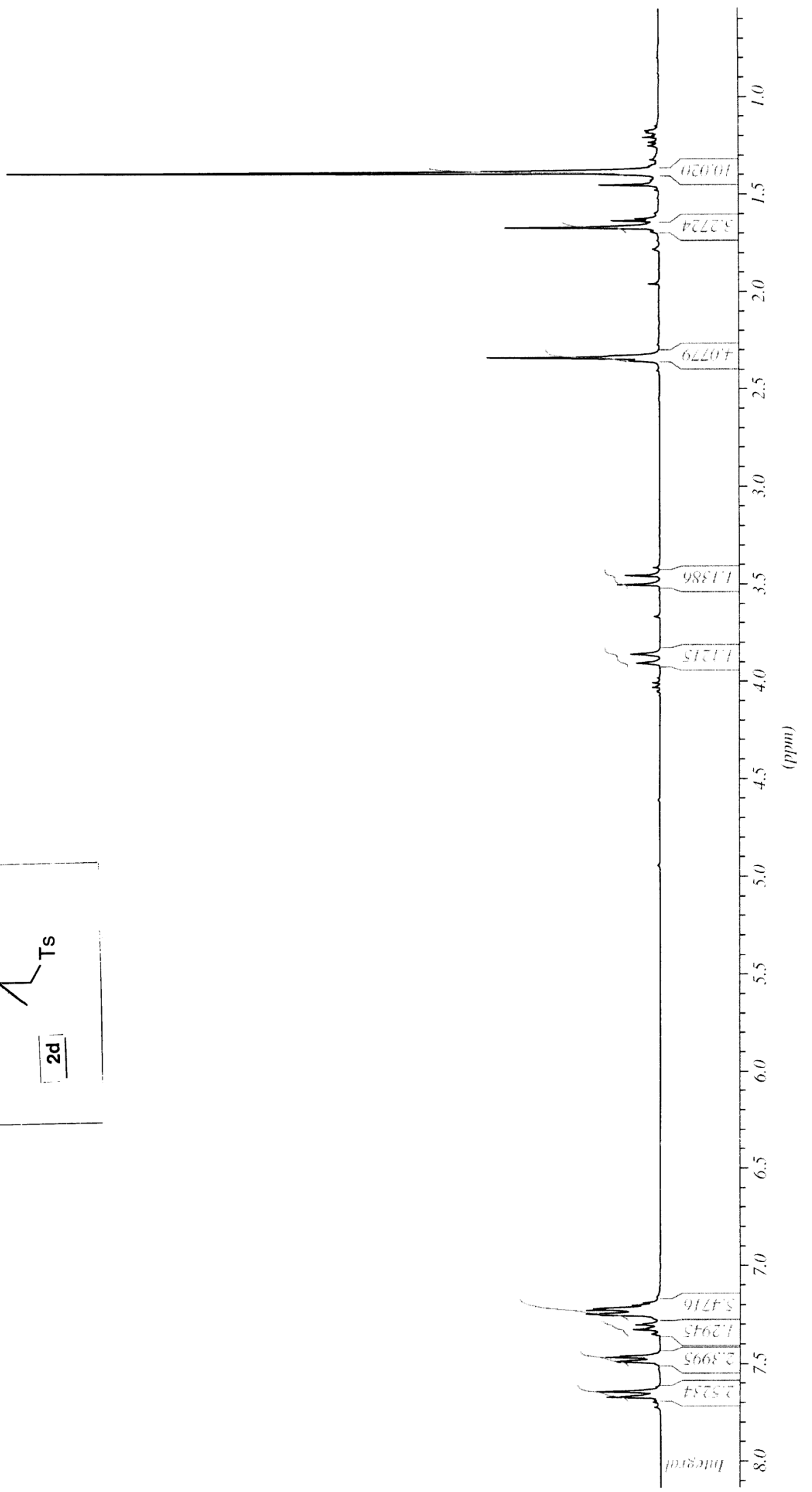


S15

$221022-2$
$99+822-$
$118182-$

$t 86 \varsigma \varsigma t$

$10<2 \varsigma 9$

9Zடะะ8-

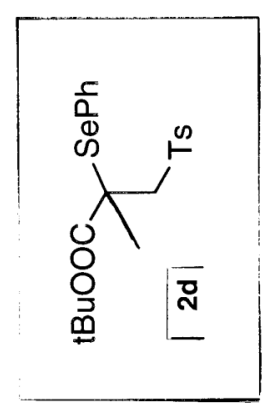

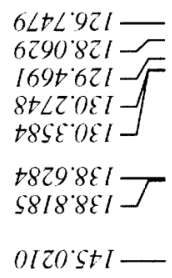

$9118^{\circ} 0 \angle 1-$

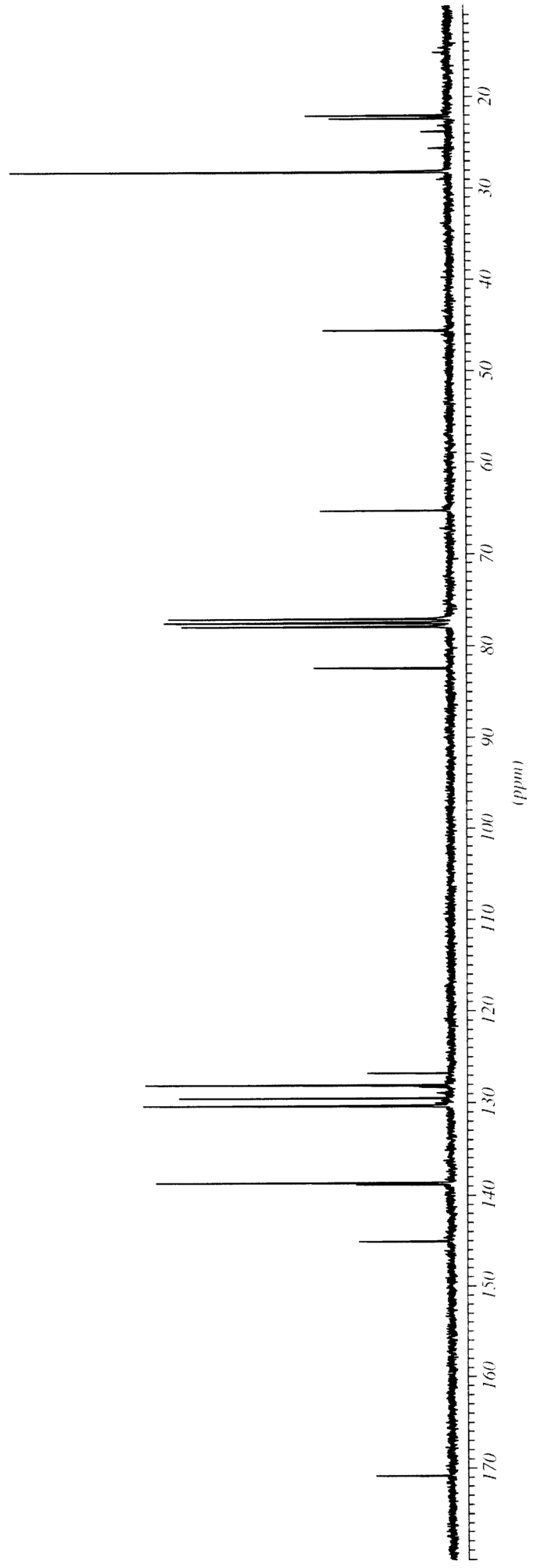


S16
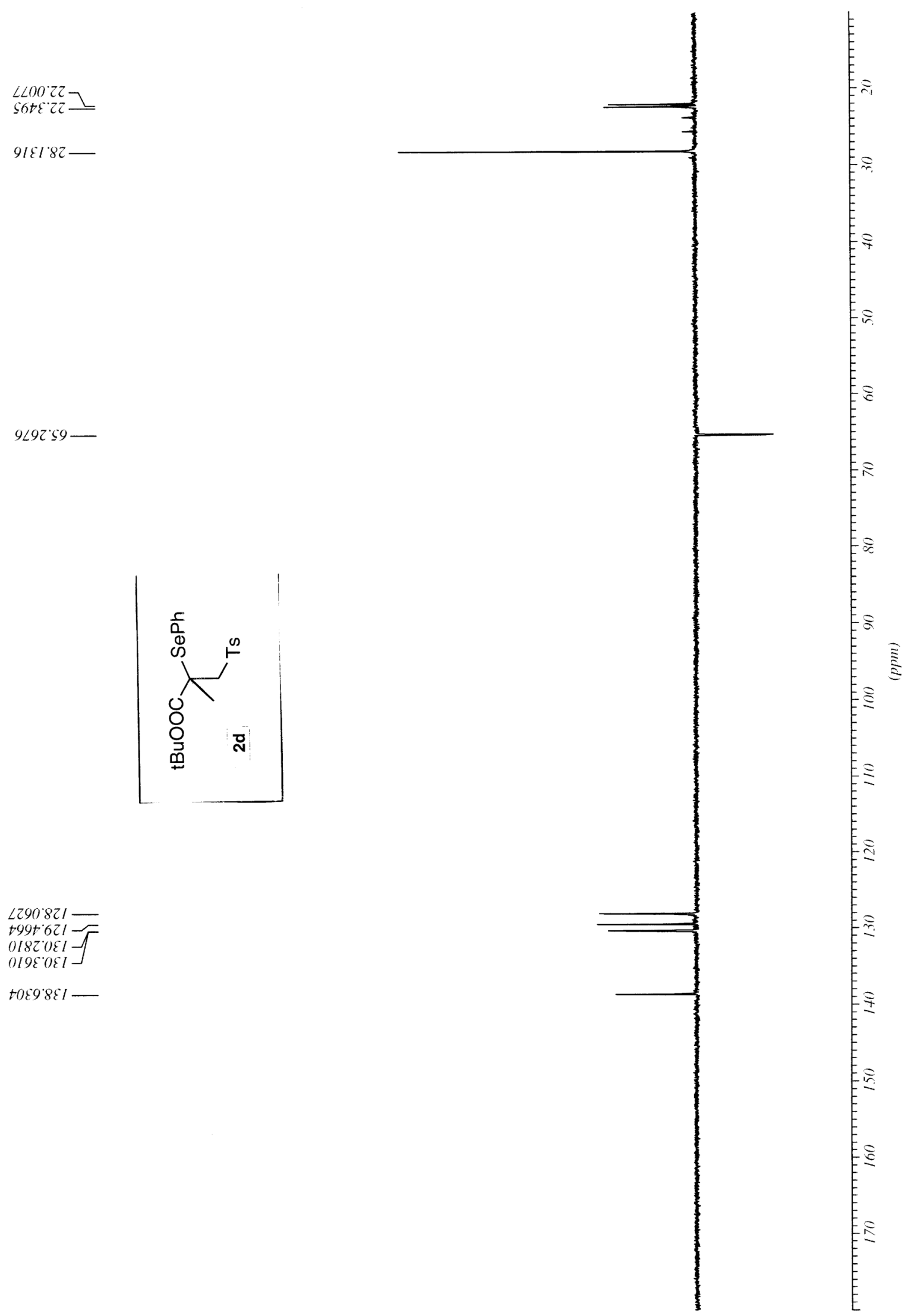

$2290821-$
$+09+621-$

OI8ZOE I-

OISZOEL

t089881-

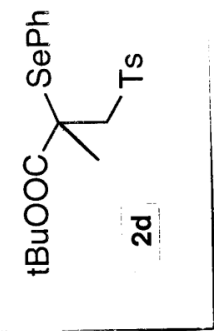


S17

$6 \varepsilon \varepsilon \angle 9$
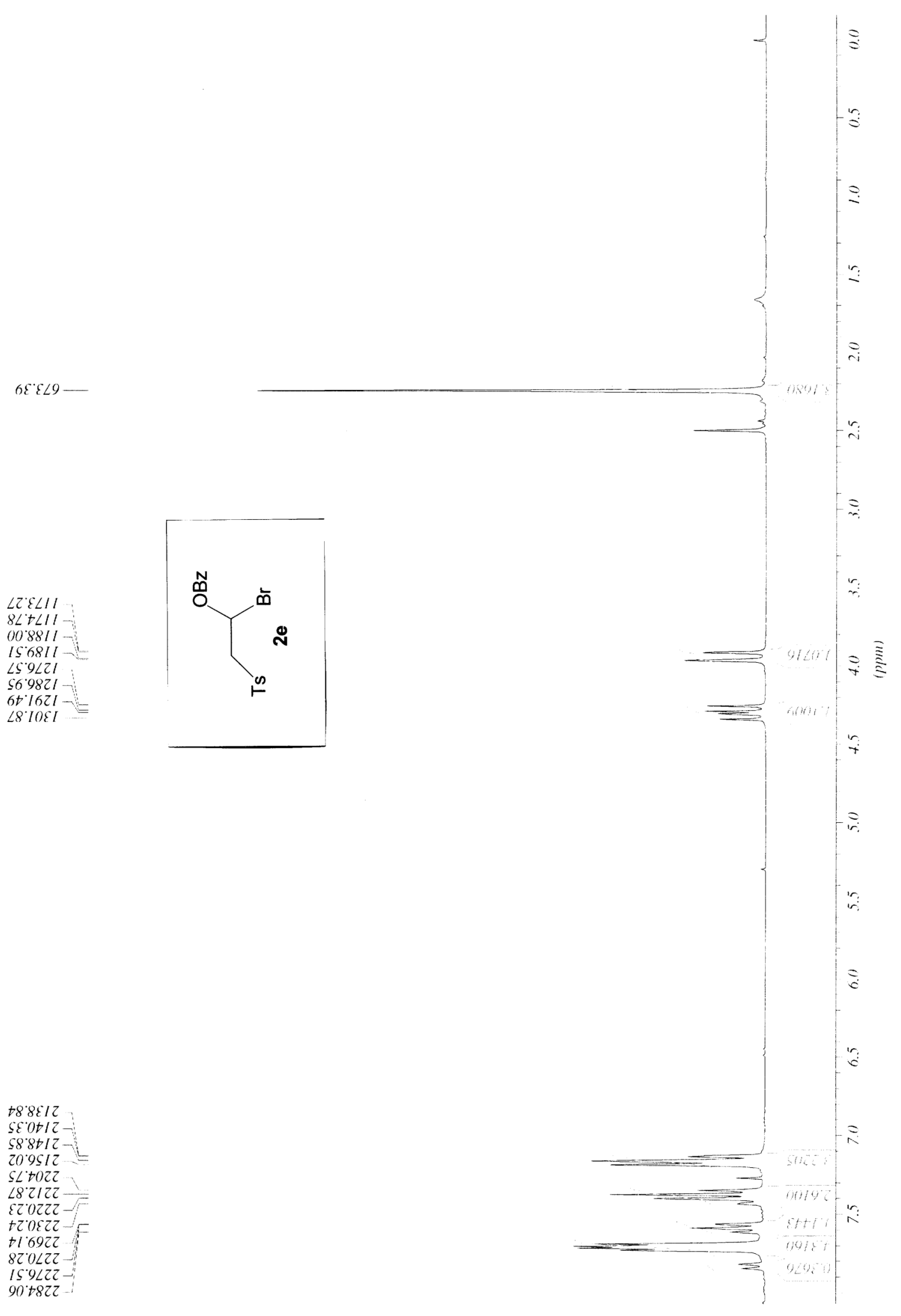

$+8.8 \varepsilon 12$

$580+12$

$588+12-$

20.9512

$s \angle t) Z 2$

$\angle 82122$

$820 z z z-1$

trogzz-

+1.69z2

$82^{\circ} 0 \angle 22$

$150<2 z-$

$90+822$ 


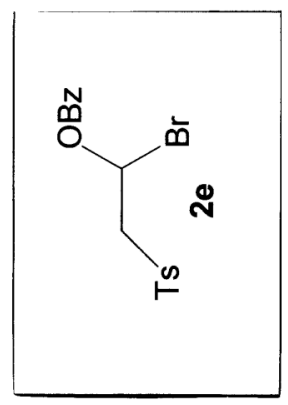

$68+6.221$
$50 \angle 0.821]$
$+8 \angle t .821]$
$229 L 821]$
$108+081=$
$1955081]$
$8 E 25081=$
$12 \angle 2.981$

$\angle 928^{\circ} S t I-$

$\angle 6 \mathcal{E} \mathcal{E} \mathcal{G} 9 I-$

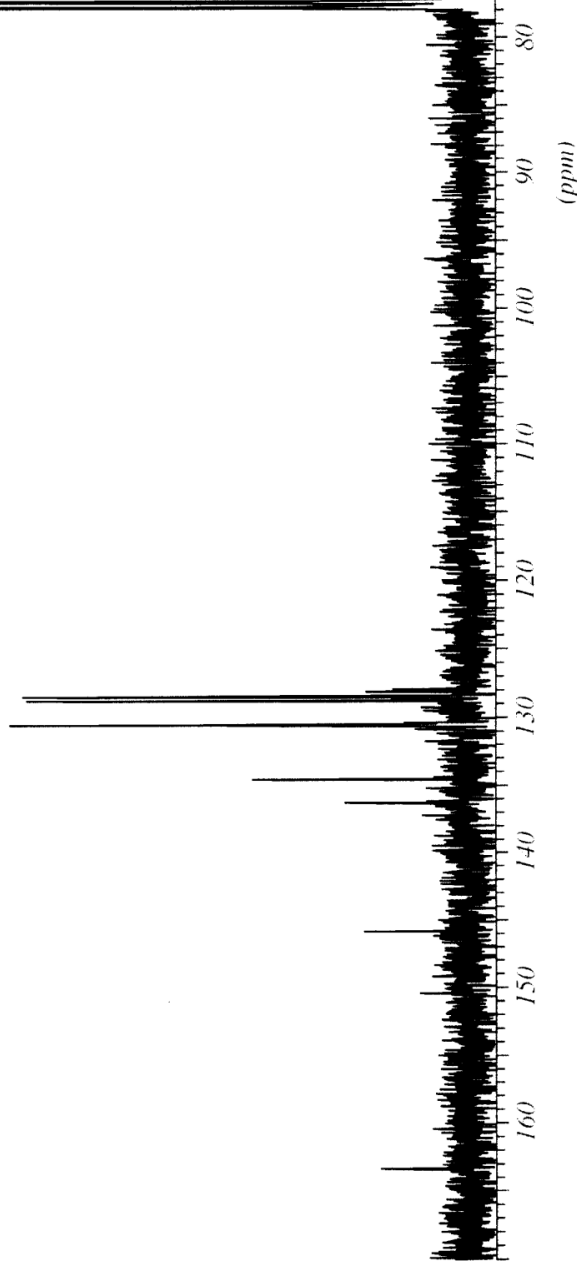


S19

$6506 \%$
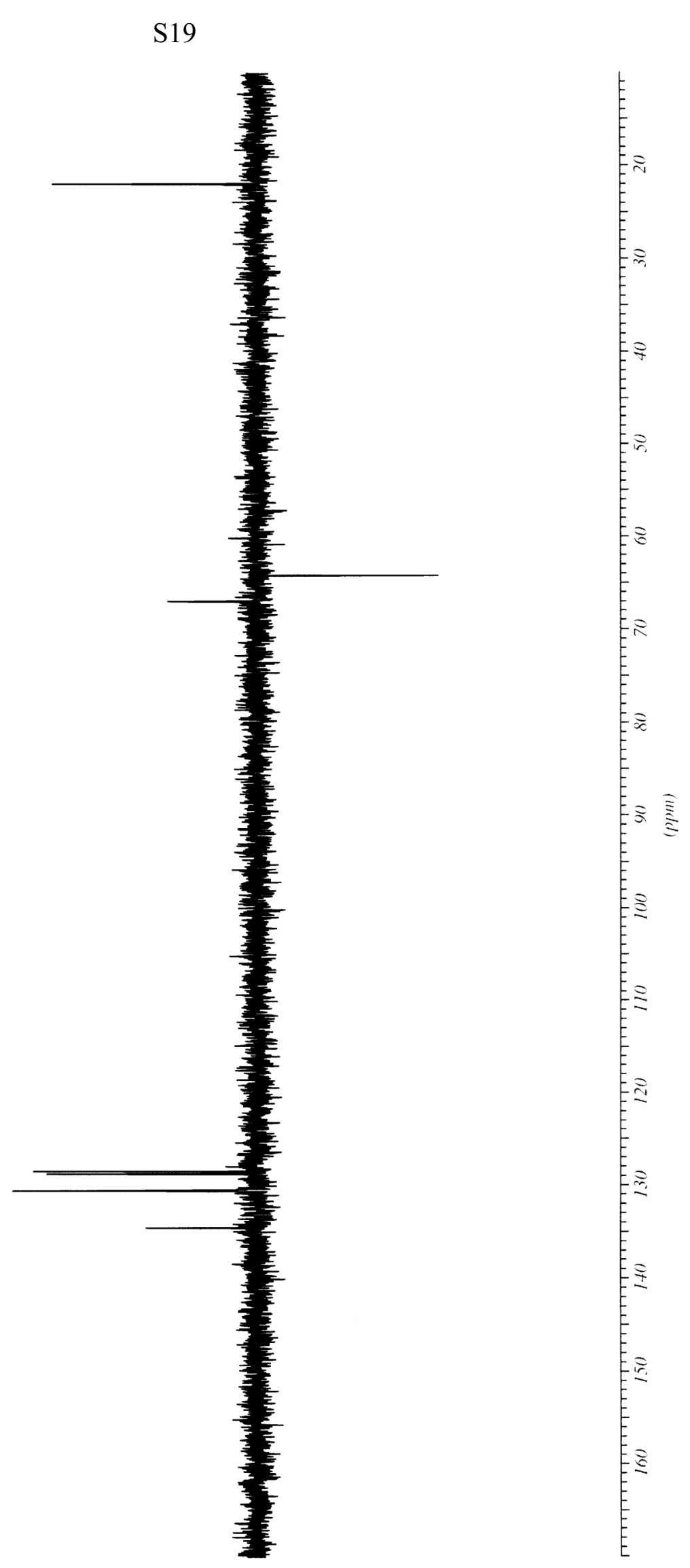

$\varepsilon \angle \angle t 821$
$609 \angle 821 \square$

$9+8+0 \varepsilon 1$

$\angle S S O E I-$

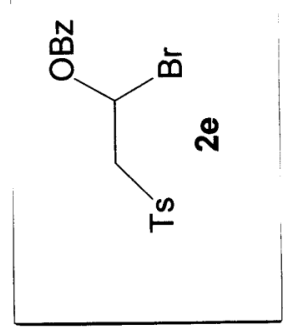

$5825+81=$ 

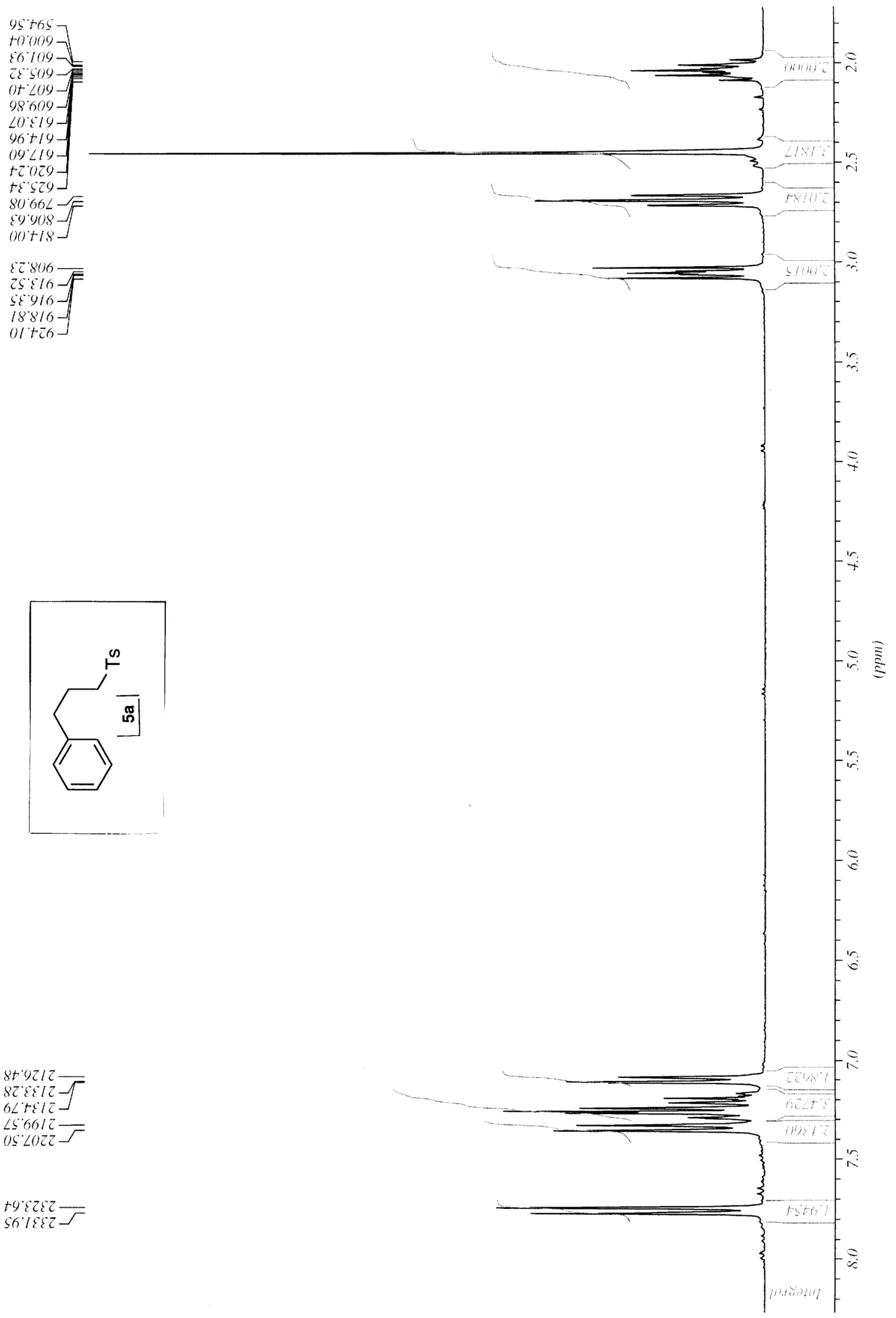
861022

$t \angle S 9+2$

t805't

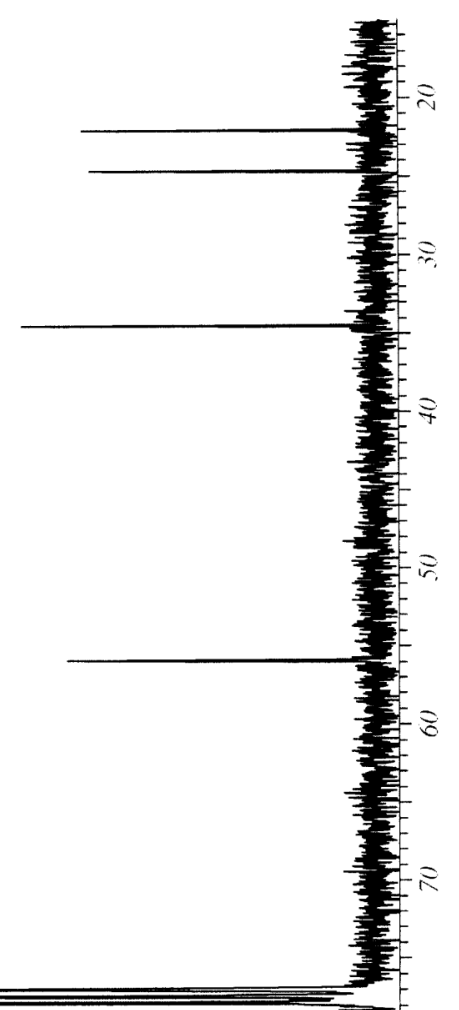

SEt6 $S \mathcal{-}$

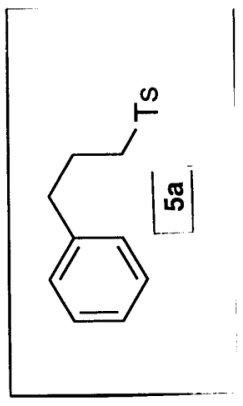

SE6L $921-$

$859+821-$

$8692821-1$
9286.821

$0286.821]$

$1885981-$

6SlEOtl-

$98205+1-$

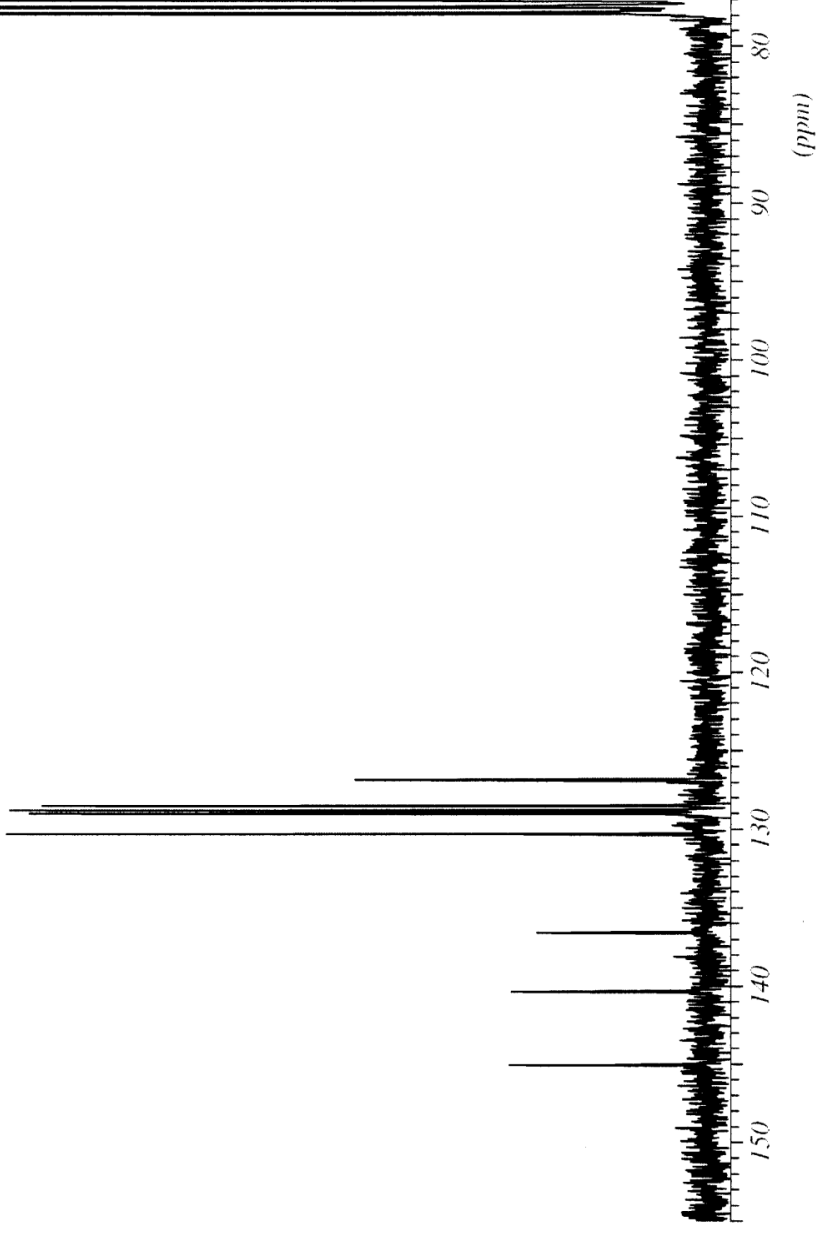


861022

$5659+2$

$6905^{\circ}+8$
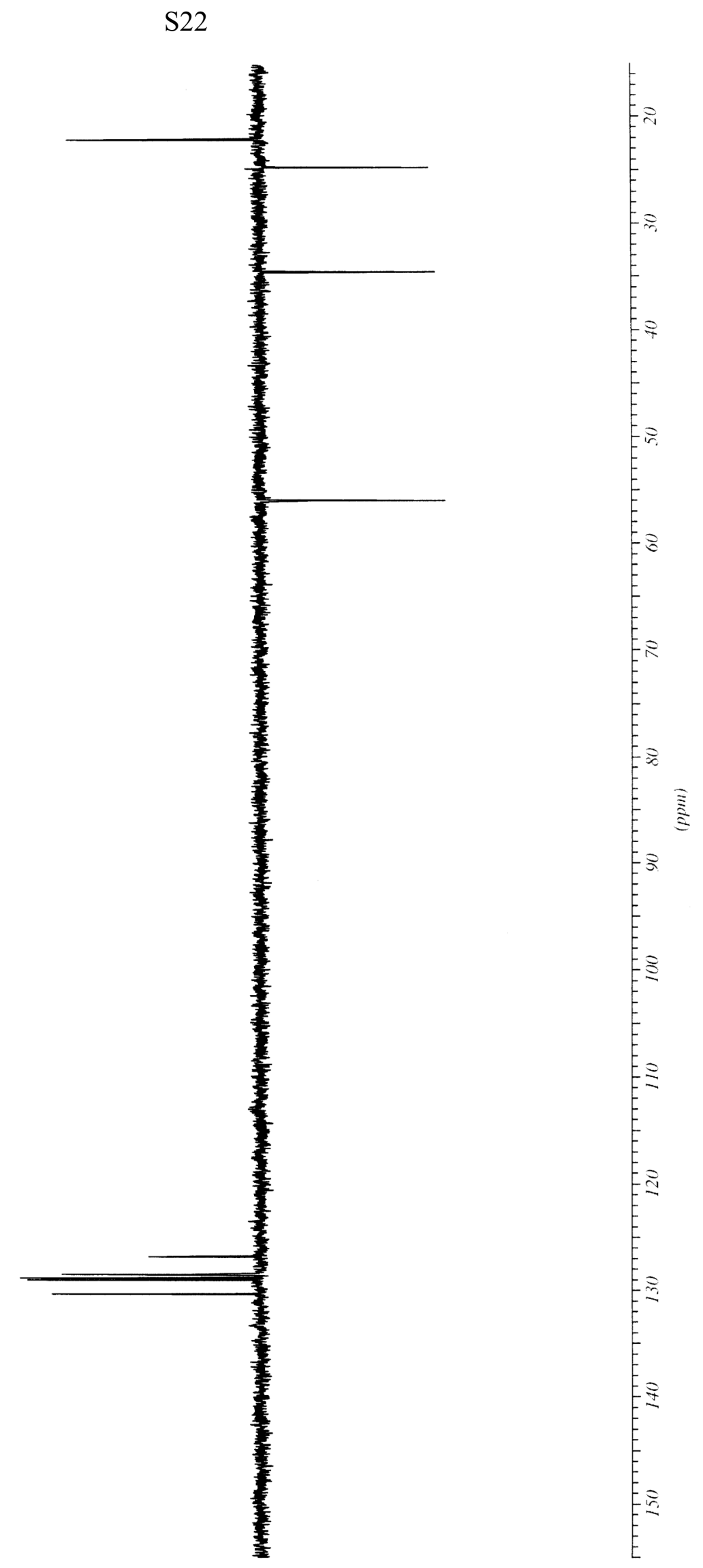

$2062921=$
$795+821=$

$+892821-$

t+ $26821-$

8082081 
$1+68+$

$0 /+5 s$

69.1957

$22+95$

58695

68089

92.989

$10.989-$

$+5889-$

$+1.969]$

$68^{\circ}+\angle L$

$69.182-\bar{E}$

$59.184-$

$+156 \mathrm{~L}$

06108
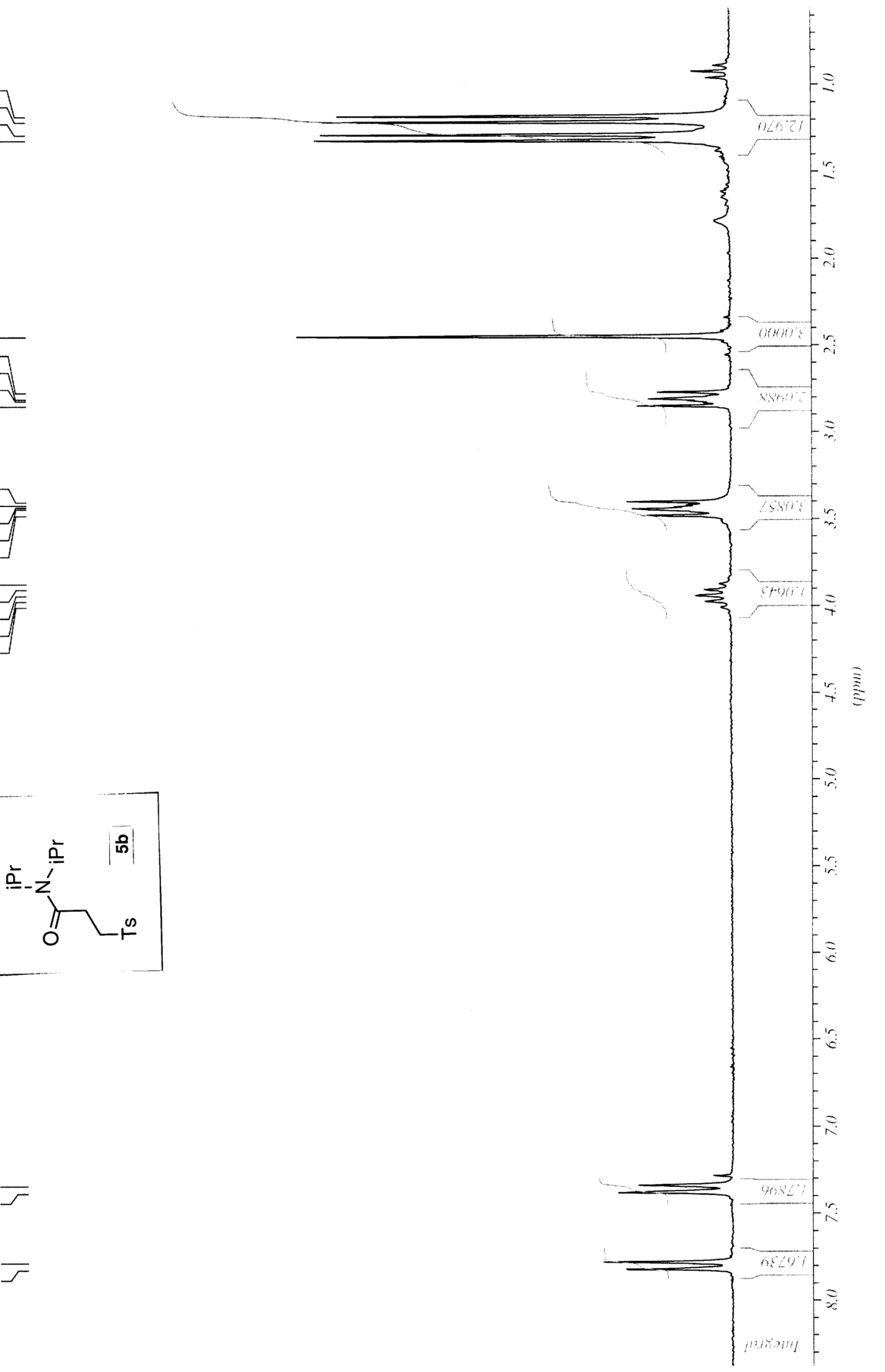

$99: 29+1-$

$\angle 6 \$ S S I$

$\varepsilon I+9 S I-$ 
$69265 t$

201485

812225

$050802-2$

$6195 \angle \vec{c}$

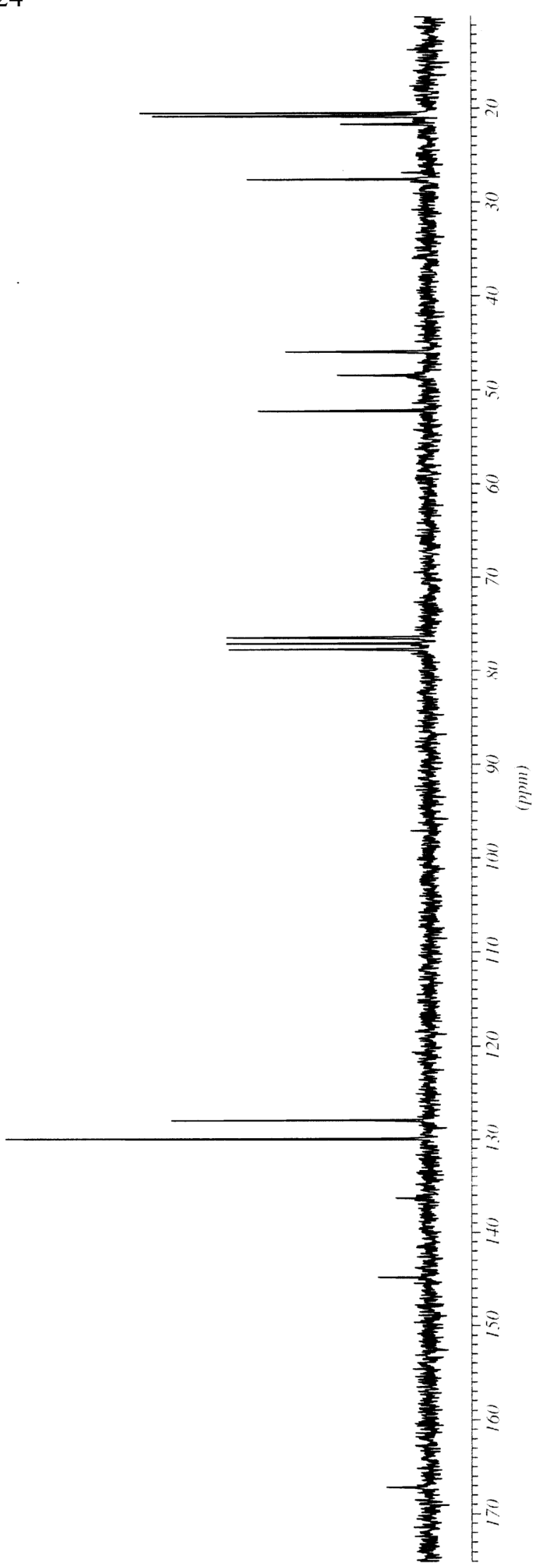

$\prod_{0}$

$6886 \circ 21-$
$5268^{\circ} \circ 21-$

$06 \angle 2981$

$589 \angle t+1$

$2 \angle+I \angle 9 I$ 

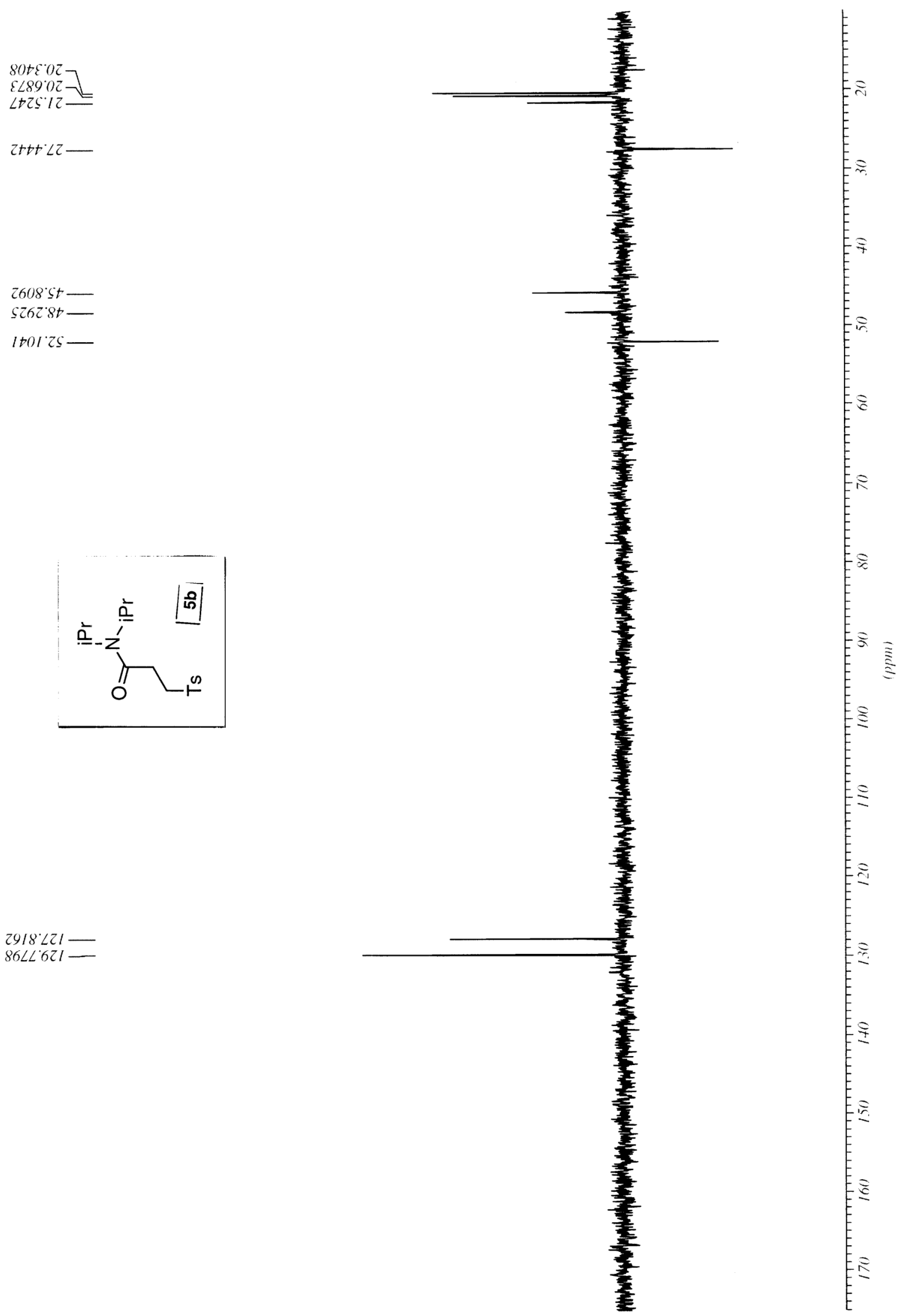

$2918.221-$
$86 \angle L 621=$ 
$9292 L-$

\section{$2+106$}

41556

58.96

58296

$5<6101-$

$\left.\begin{array}{l}16.2201 \\ 89.2201\end{array}\right]=$

28.8201

$1 \angle 0801]$

959801 트

\section{(⿻)}

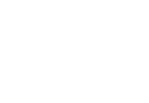
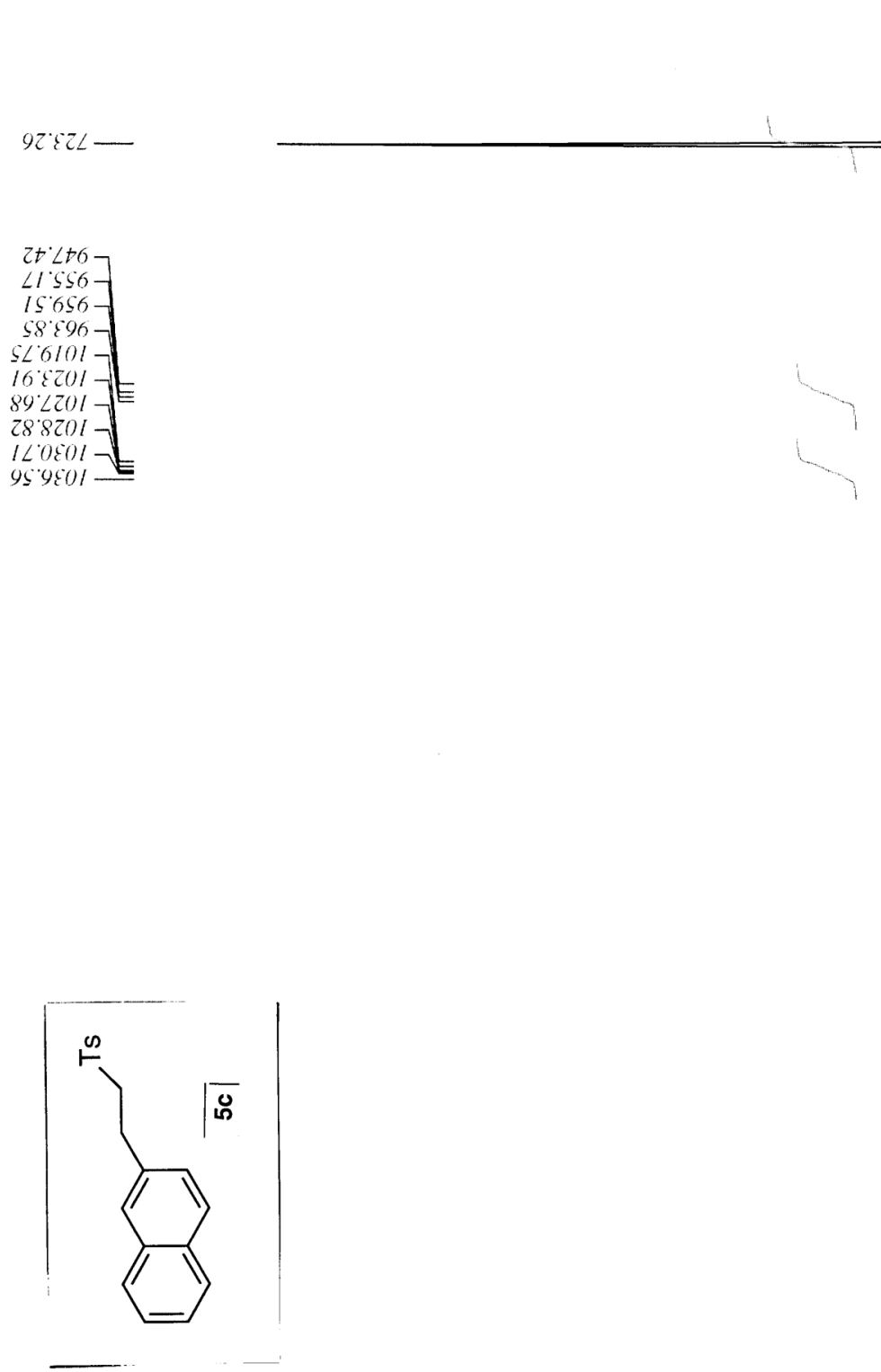

19.9512

$862512-$

$80.9912-$

89.2612 1

$10.0028 \mathrm{Z}$

$190022=$

tL8122-原

$+8{ }^{\circ} \mathrm{czz}-$

ts seze-

os $6 z 2 z-1=$

$8 Z^{\circ} \varepsilon \varepsilon z 2-$

$800022-1$

$128522-$

is $6082-$

$26.818 z$ 
$t \angle 20: z z-$

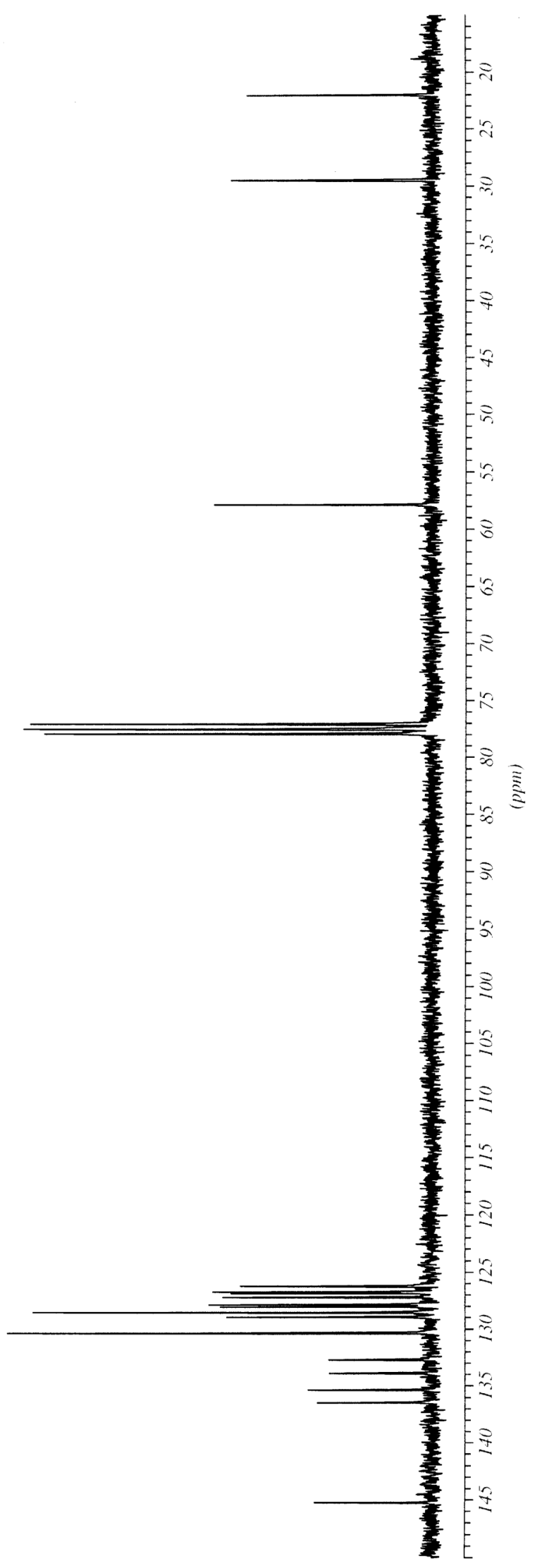

9002921

$\$ \angle 1<921$

otor.221

$1058<21$

LLt082I I

9925821

$9+56821]$

099802

$+59^{\circ} 2 \mathrm{CLI}$

$20 \angle 8 \% 81=F$

9628981

$88225+1-$ 

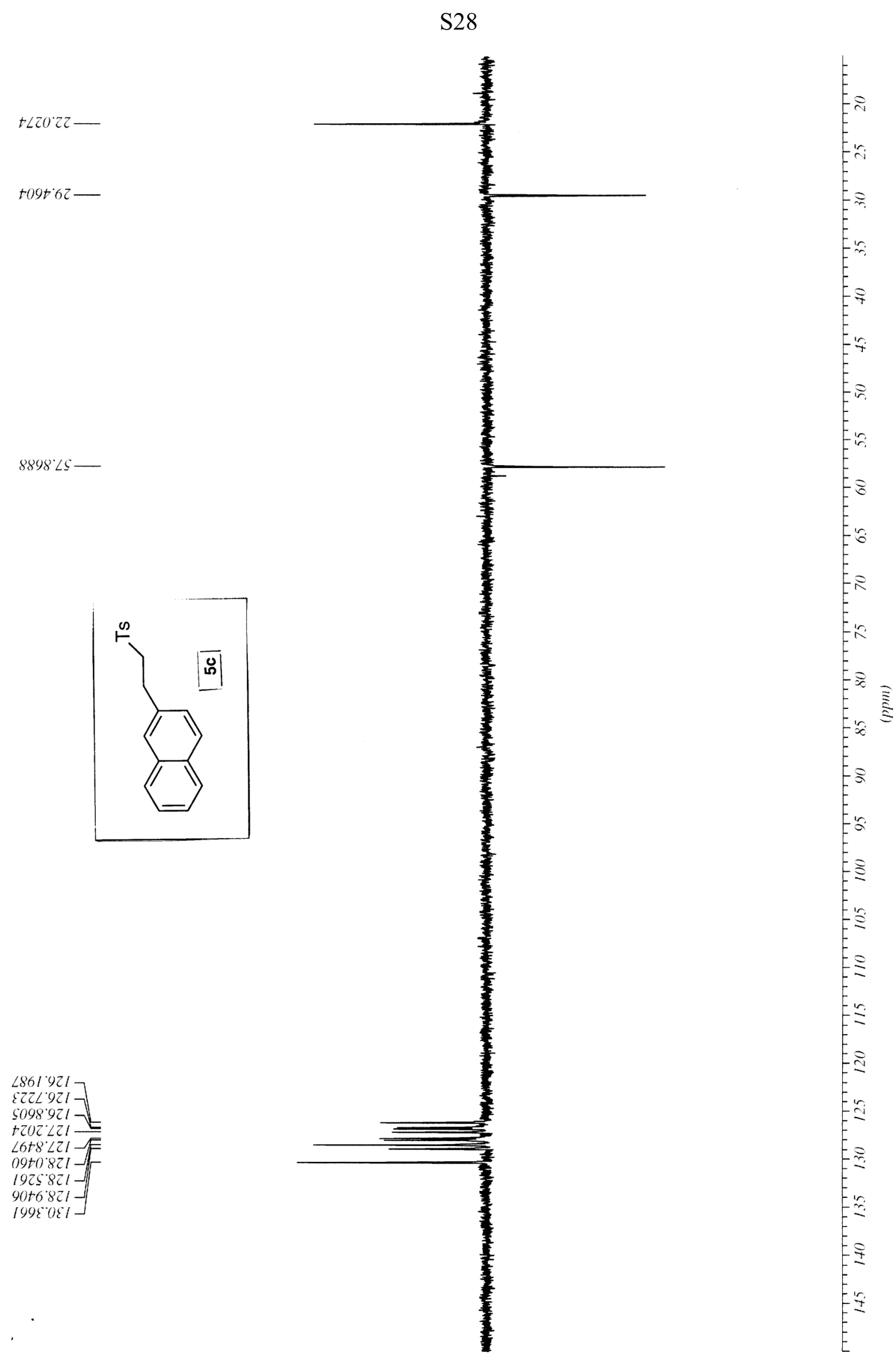


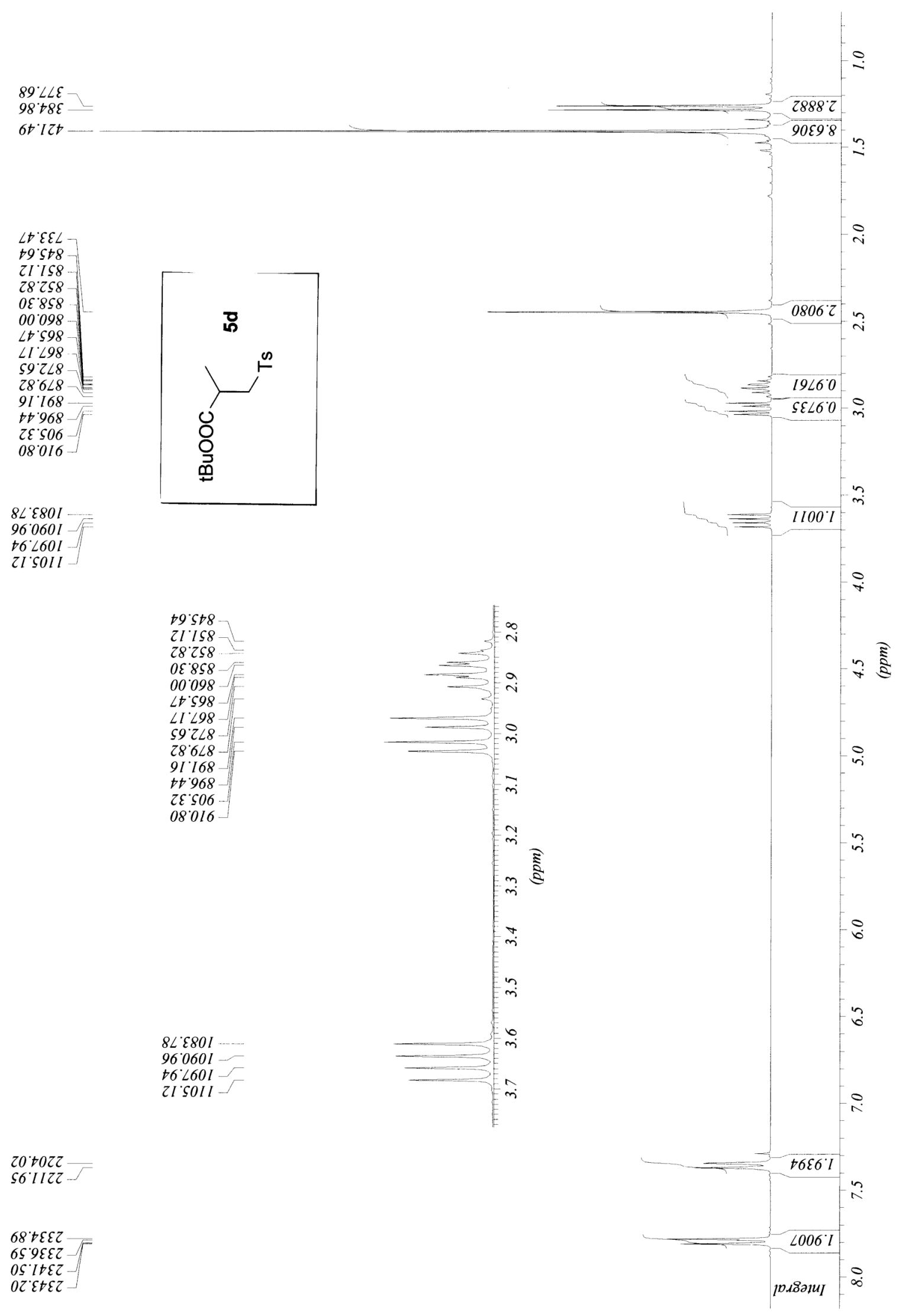




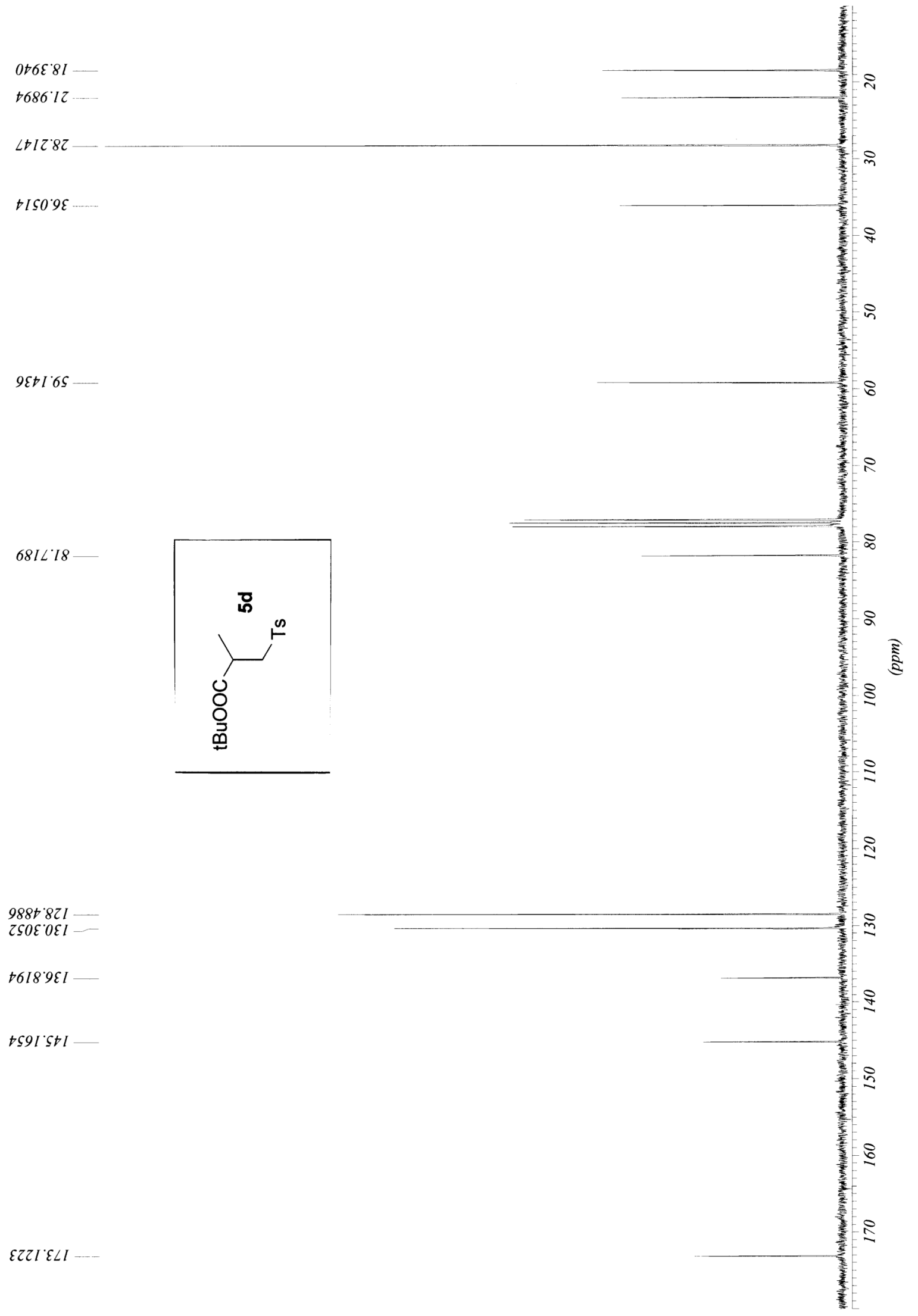



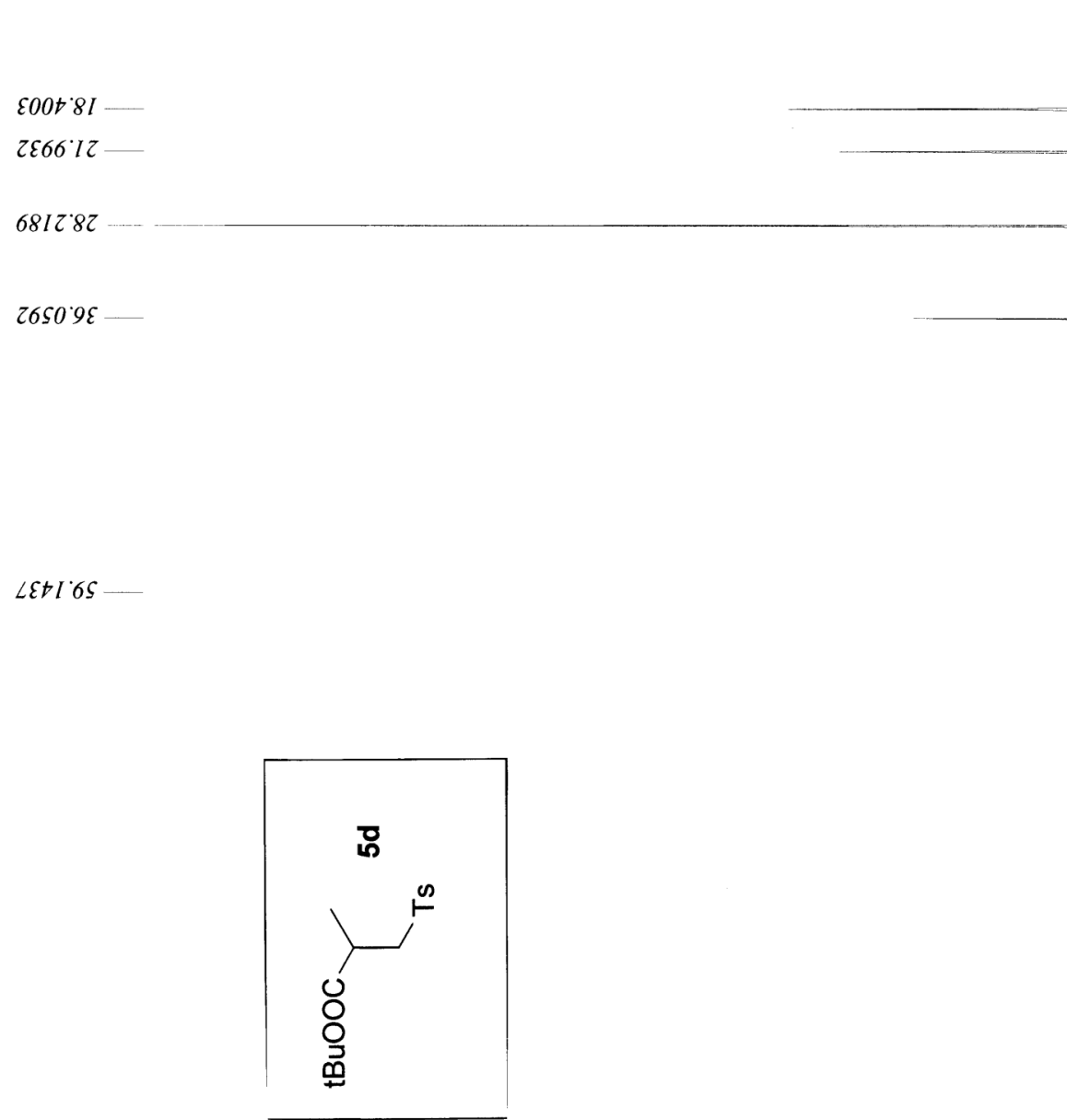

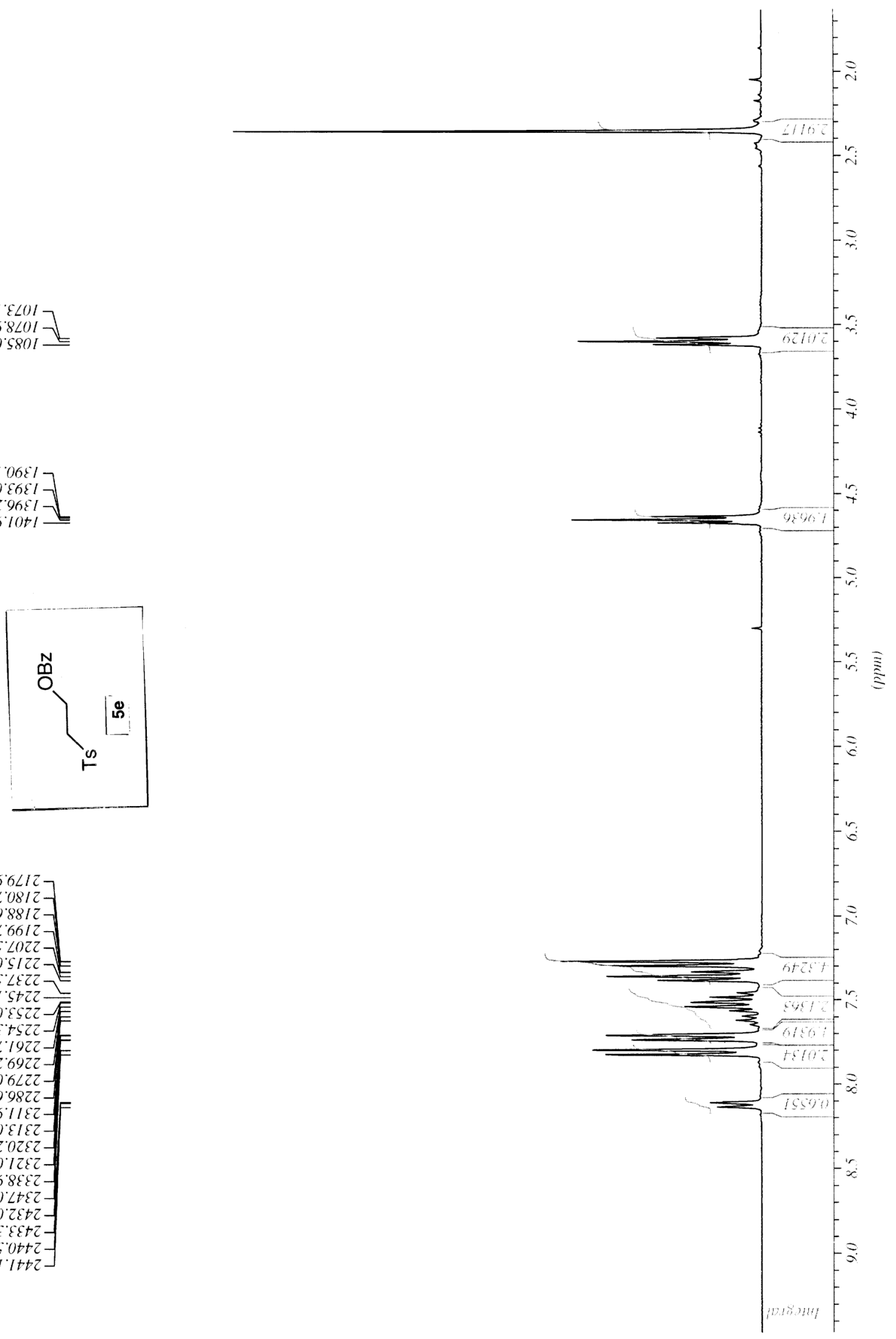

5604127

$5688 \varepsilon 2-$

$80^{\circ} \angle t \varepsilon z-$

$90^{\circ} \mathrm{cstz}-$

$8 \varepsilon^{\circ} \varepsilon \mathcal{E}+\tau$

$s S^{\circ} 0 t+2-$

$z I+1+t z$ 
S33

065612

$\varsigma \angle \angle 9^{\circ} S \varsigma-$

095285-

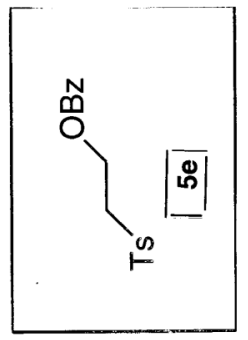

$988+821$

2019821

$29 \angle 8.821$

$880+621-$

$0+20081-$

EILSOEI -

$1089 \mathrm{gEI}-$

zorest

$20+\varepsilon s+1-$

EL91.991 -

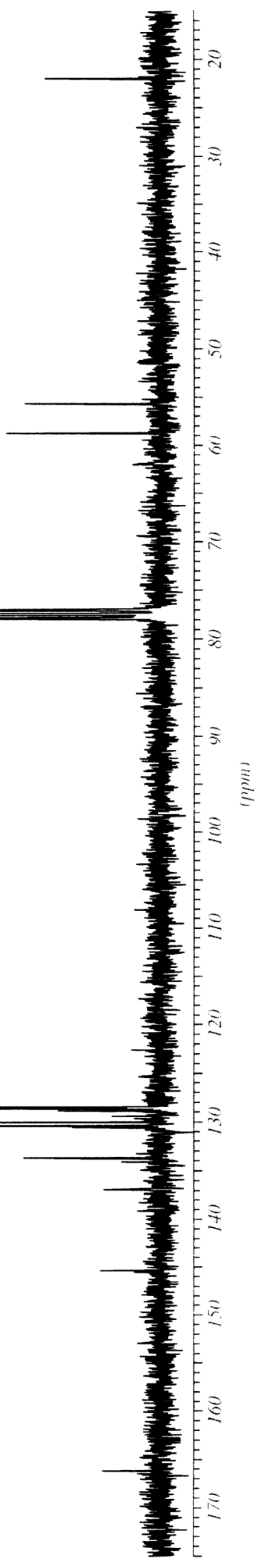


It96:IZ-
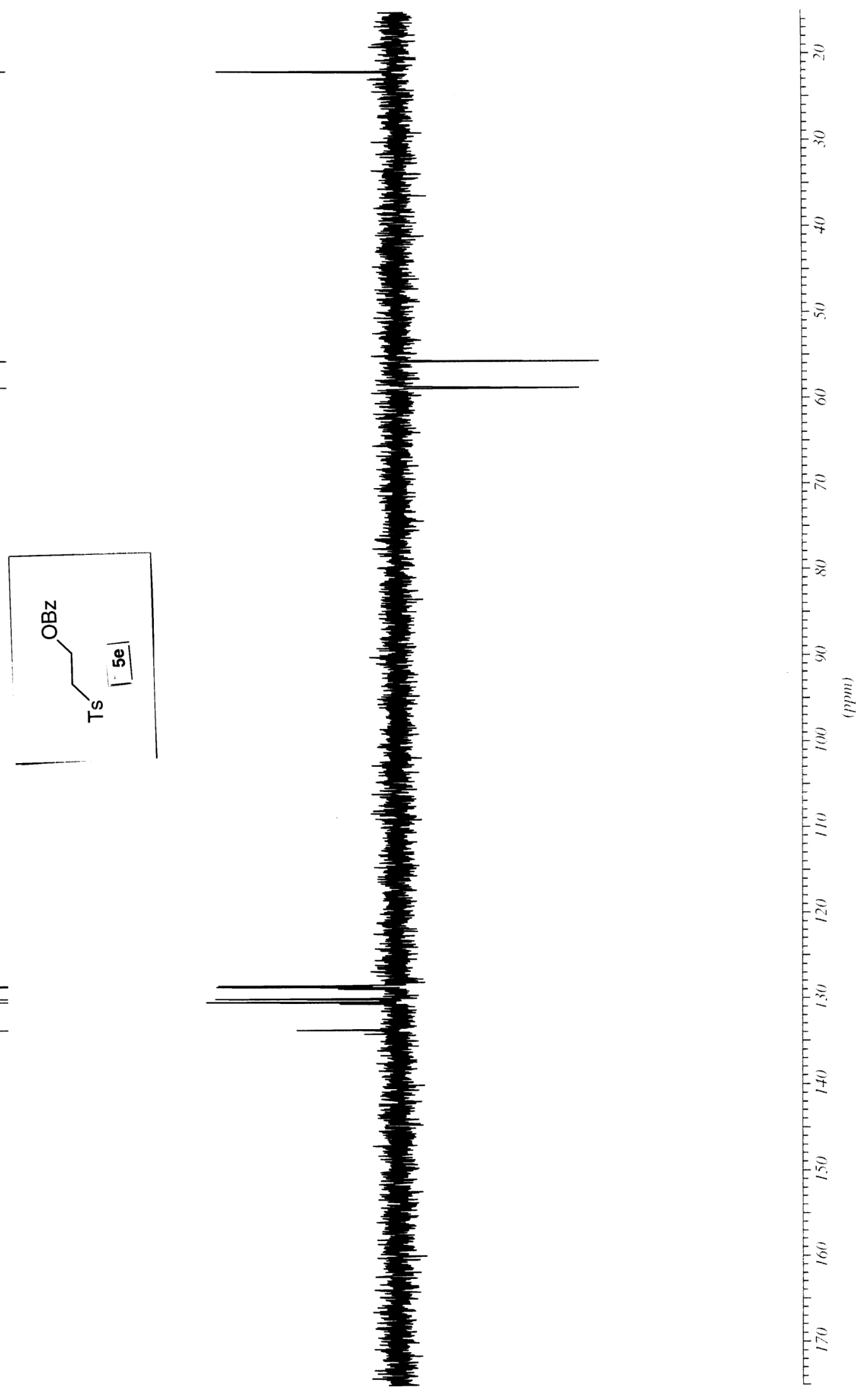

$816 t^{\circ} 821$
$2809.821]$

t920 $0 \mathrm{E} I-$

$9+0 t 081-$
$8+80^{\circ} .81$ 
Table S1. kinetic experiments with compound 2a

\begin{tabular}{lllll}
\hline $\mathrm{T}, \mathrm{K}$ & {$\left[\mathrm{Bu}_{3} \mathrm{SnH}\right]_{0}, \mathrm{M}$} & {$[\mathbf{5}] /[\mathbf{4}]$} & $\mathrm{k}_{\mathrm{H}} / \mathrm{k}_{\mathrm{f}}, \mathrm{M}^{-1}$ & intercept \\
\hline 293 & 0.398 & 0.53 & $1.0 \pm 0.1$ & $0.13 \pm 0.01$ \\
& 0.513 & 0.651 & & \\
& 0.62 & 0.756 & & \\
\hline
\end{tabular}

Table S2. kinetic experiments with compound 2a'

\begin{tabular}{lllll}
\hline $\mathrm{T}, \mathrm{K}$ & {$\left[\mathrm{Bu}_{3} \mathrm{SnH}\right]_{0}, \mathrm{M}$} & {$[\mathbf{5}] /[4]$} & $\mathrm{k}_{\mathrm{H}} / \mathrm{k}_{\mathrm{f}}, \mathrm{M}^{-1}$ & intercept \\
\hline 293 & 0.108 & 0.221 & $1.6 \pm 0.2$ & $0.06 \pm 0.04$ \\
& 0.21 & 0.426 & & \\
& 0.275 & 0.479 & & \\
& 0.338 & 0.594 & & \\
\hline
\end{tabular}

Table S3. kinetic experiments with compound 2c

\begin{tabular}{lllll}
\hline $\mathrm{T}, \mathrm{K}$ & {$\left[\mathrm{Bu}_{3} \mathrm{SnH}\right]_{0}, \mathrm{M}$} & {$[5] /[4]$} & $\mathrm{k}_{\mathrm{H}} / \mathrm{k}_{\mathrm{f}}, \mathrm{M}^{-1}$ & intercept \\
\hline 403 & 0.037 & 0.029 & $0.26 \pm 0.01$ & $0.017 \pm 0.004$ \\
& 0.108 & 0.048 & & \\
& 0.21 & 0.063 & & \\
& 0.338 & 0.104 & & \\
& 0.62 & 0.179 & & \\
\hline
\end{tabular}

Table S4. kinetic experiments with compound 2d

\begin{tabular}{lllll}
\hline $\mathrm{T}, \mathrm{K}$ & {$\left[\mathrm{Bu}_{3} \mathrm{SnH}\right]_{0}, \mathrm{M}$} & {$[\mathbf{5}] /[\mathbf{4}]$} & $\mathrm{k}_{\mathrm{H}} / \mathrm{k}_{\mathrm{f}}, \mathrm{M}^{-1}$ & intercept \\
\hline 293 & 0.0074 & 0.968 & $159 \pm 39$ & $0.002 \pm 0.53$ \\
& 0.011 & 2.13 & & \\
& 0.015 & 2.27 & & \\
& 0.018 & 2.83 & & \\
\hline
\end{tabular}

Table S5. kinetic experiments with compound 2e

\begin{tabular}{lllll}
\hline $\mathrm{T}, \mathrm{K}$ & {$\left[\mathrm{Bu}_{3} \mathrm{SnH}\right]_{0}, \mathrm{M}$} & {$[\mathbf{5}] /[\mathbf{4}]$} & $\mathrm{k}_{\mathrm{H}} / \mathrm{k}_{\mathrm{f}}, \mathrm{M}^{-1}$ & intercept \\
\hline 293 & 0.091 & 0.24 & $4.4 \pm 0.4$ & $-0.14 \pm 0.07$ \\
& 0.126 & 0.42 & & \\
& 0.177 & 0.62 & & \\
& 0.189 & 0.77 & & \\
& 0.256 & 0.96 & & \\
\hline
\end{tabular}


Table S6. kinetic experiments with compound $\mathbf{2 b}$

\begin{tabular}{|c|c|c|c|c|}
\hline $\mathrm{T}, \mathrm{K}$ & {$\left[\mathrm{Bu}_{3} \mathrm{SnH}\right]_{0}, \mathrm{M}$} & {$[5] /[4]$} & $\mathrm{k}_{\mathrm{H}} / \mathrm{k}_{\mathrm{f}}, \mathrm{M}^{-1}$ & intercept \\
\hline \multirow[t]{3}{*}{293} & 0.0028 & 0.861 & $325 \pm 47$ & $-0.04 \pm 0.17$ \\
\hline & 0.0037 & 1.2 & & \\
\hline & 0.0041 & 1.27 & & \\
\hline \multirow[t]{4}{*}{353} & 0.03 & 0.351 & $19.8 \pm 1.5$ & $-0.29 \pm 0.08$ \\
\hline & 0.044 & 0.539 & & \\
\hline & 0.059 & 0.906 & & \\
\hline & 0.073 & 1.18 & & \\
\hline \multirow[t]{4}{*}{373} & 0.03 & 0.183 & $10.6 \pm 0.5$ & $-0.16 \pm 0.04$ \\
\hline & 0.066 & 0.524 & & \\
\hline & 0.094 & 0.812 & & \\
\hline & 0.129 & 1.24 & & \\
\hline \multirow[t]{4}{*}{393} & 0.03 & 0.128 & $7.5 \pm 1.1$ & $-0.13 \pm 0.08$ \\
\hline & 0.059 & 0.233 & & \\
\hline & 0.087 & 0.572 & & \\
\hline & 0.115 & 0.725 & & \\
\hline \multirow[t]{4}{*}{410} & 0.101 & 0.156 & $3.2 \pm 0.8$ & $-0.18 \pm 0.09$ \\
\hline & 0.115 & 0.19 & & \\
\hline & 0.129 & 0.21 & & \\
\hline & 0.143 & 0.3 & & \\
\hline
\end{tabular}

